\title{
Bismuth and Titanium Phosphinates: Isolation of Tetra-, Hexa- and Octanuclear Clusters
}

Junaid Alia ${ }^{\mathrm{a}}$ Tokala Navaneetha ${ }^{\mathrm{a}}$, Viswanathan Baskar ${ }^{{ }^{*}}$

School of Chemistry, University of Hyderabad Hyderabad 500046, Telangana, India.

E-mail: vbsc@uohyd.ac.in.

\section{Contents:}

1. Table S1. Crystallographic information of compounds 1-4.

2. Table S2. Selected bond lengths $(\AA)$ and Bond angles (deg) parameters of compound $\mathbf{1}$.

3. Table S3. Selected bond lengths $(\AA)$ and Bond angles (deg) parameters of compound 2.

4. Table S4. Selected bond lengths (Å) and Bond angles (deg) parameters of compound 3.

5. Table S5. Selected bond lengths $(\AA)$ and Bond angles (deg) parameters of compound 4.

6. Table S6. Shape calculation of compound 1 .

7. Table S7. Shape calculation of compound 2.

8. Figure S1. ORTEP view of $\mathbf{1}$.

9. Figure S2. Solution ${ }^{31} \mathrm{P}$ NMR spectrum of $\mathbf{1}$.

10. Figure S3. Solution ${ }^{1} \mathrm{H}$ NMR spectrum of 1

11. Figure S4. Solution ${ }^{13} \mathrm{C}$ NMR spectrum of $\mathbf{1}$.

12. Figure S5. Powder XRD of compound 1.

13. Figure S6. TGA of compound 1.

14. Figure S7. IR spectrum of compound 1.

15. Figure S8. ORTEP view of 2.

16. Figure S9. Solution ${ }^{31} \mathrm{P}$ NMR spectrum of 2 .

17. Figure S10. Solution ${ }^{1} \mathrm{H}$ NMR spectrum of 2.

18. Figure S11. Solution ${ }^{13} \mathrm{C}$ NMR spectrum of 2 .

19. Figure S12. Powder XRD of compound 2.

20. Figure S13. TGA of compound 2.

21. Figure S14. IR spectrum of compound 2.

22. Figure S15. ORTEP view of 3 .

23. Figure S16. Solution ${ }^{31} \mathrm{P}$ NMR spectrum of 3 .

24. Figure S17. Solution ${ }^{1} \mathrm{H}$ NMR spectrum of 3 .

25. Figure S18. Solution ${ }^{13} \mathrm{C}$ NMR spectrum of 3 .

26. Figure S19. Powder XRD of compound 3 .

27. Figure S20. TGA of compound 3.

28. Figure S21. IR spectrum of compound 3 .

29. Figure S22. ORTEP view of 4.

30. Figure S23. Solution ${ }^{31} \mathrm{P}$ NMR spectrum of 4 at $25^{\circ} \mathrm{C}$.

31. Figure S24. Solution ${ }^{31} \mathrm{P}$ NMR spectrum of 4 at $0^{\circ} \mathrm{C}$.

32. Figure S25. Solution ${ }^{31} \mathrm{P}$ NMR spectrum of 4 at $-10^{\circ} \mathrm{C}$.

33. Figure S26. Solution ${ }^{31} \mathrm{P}$ NMR spectrum of 4 at $-20^{\circ} \mathrm{C}$. 
34. Figure S27. Solution ${ }^{31} \mathrm{P}$ NMR spectrum of 4 at $-30^{\circ} \mathrm{C}$.

35. Figure S28. Solution ${ }^{31} \mathrm{P}$ NMR spectrum of 4 at $-40^{\circ} \mathrm{C}$.

36. Figure S29. Solution ${ }^{1} \mathrm{H}$ NMR spectrum of $\mathbf{4}$.

37. Figure S30. Solution ${ }^{13} \mathrm{C}$ NMR spectrum of 4.

38. Figure S31. Powder XRD of compound 4.

39. Figure S32. TGA of compound 4.

40. Figure S33. IR spectrum of compound 4.

41. Figure S34. Packing diagram of compound 1.

42. Figure S35. Packing diagram of compound 2.

43. Figure S36. Packing diagram of compound 3.

44. Figure S37. Packing diagram of compound 4.

45. Figure S38. Powder X-ray diffraction pattern of the residue sample of $\mathbf{1}$.

46. Figure S39. Powder X-ray diffraction pattern of the residue sample of 2.

47. Figure S40. Powder X-ray diffraction pattern of the residue sample of $\mathbf{3}$.

48. Figure S41. Powder X-ray diffraction pattern of the residue sample of 4 . 
Table S1: crystallographic information of compounds 1-4.

\begin{tabular}{|c|c|c|c|c|}
\hline & 1 & 2 & 3 & 4 \\
\hline Formula & $\mathrm{C}_{59} \mathrm{H}_{52.5} \mathrm{Bi}_{1} \mathrm{~N}_{1.5} \mathrm{O}_{4.5} \mathrm{P}_{2}$ & $\mathrm{C}_{155} \mathrm{H}_{137.5} \mathrm{Bi}_{4} \mathrm{Cl}_{4} \mathrm{~N}_{0.5} \mathrm{O}_{20} \mathrm{P}_{8}$ & $\mathrm{C}_{249} \mathrm{H}_{220} \mathrm{O}_{36} \mathrm{P}_{12} \mathrm{Ti}_{8}$ & $\mathrm{C}_{80} \mathrm{H}_{158} \mathrm{O}_{31} \mathrm{P}_{9} \mathrm{Ti}_{6} \mathrm{~N}_{4}$ \\
\hline F.wt $\mathrm{g} / \mathrm{mol}^{-1}$ & 1125.44 & 3552.63 & 4543.08 & 2238.22 \\
\hline $\mathrm{T}, \mathrm{K}$ & $296(2)$ & $100(2)$ & $100(2)$ & $100(2)$ \\
\hline Crystal system & Triclinic & Monoclinic & Triclinic & Orthorhombic \\
\hline Space group & $P-1$ & $\mathrm{P} 21 / \mathrm{n}$ & $P-1$ & Pnma \\
\hline Crystal size $\mathrm{mm}^{3}$ & $0.25 \times 0.21 \times 0.18$ & $0.2 \times 0.16 \times 0.12$ & $0.19 \times 0.15 \times 0.12$ & $0.2 \times 0.18 \times 0.15$ \\
\hline a, $\AA$ & $12.2655(13)$ & $17.9800(2)$ & $19.5800(14)$ & $23.2426(9)$ \\
\hline$b, \AA$ & $13.9055(13)$ & $42.7312(6)$ & $19.8398(16)$ & $19.9079(8)$ \\
\hline c, $\AA$ & $15.0308(16)$ & $18.1373(3)$ & $19.9925(17)$ & $25.9479(11)$ \\
\hline$\alpha /^{\circ}$ & $79.116(4)$ & 90 & $61.002(3)$ & 90 \\
\hline$\beta /^{\circ}$ & $88.355(4)$ & $90.059(1)$ & $61.107(3)$ & 90 \\
\hline$r 1^{\circ}$ & $87.461(4)$ & 90 & $76.424(4)$ & 90 \\
\hline $\mathrm{V}, \AA ̊ \AA 3$ & $2514.5(4)$ & $13935.0(3)$ & $5947.2(9)$ & $12006.4(8)$ \\
\hline $\bar{Z}$ & 2 & 4 & 1 & 4 \\
\hline $\mathrm{D}_{\text {calcd }} \mathrm{Mg} / \mathrm{m3}$ & 1.486 & 1.693 & 1.268 & 1.238 \\
\hline$\mu, \mathrm{mm}-1$ & 3.620 & 5.274 & 0.403 & 0.565 \\
\hline$F(000)$ & 1132.0 & 7004.0 & 2358.0 & 4724.0 \\
\hline 2 Theta range, deg & 5.066 to 51.658 & 3.192 to 52.964 & 4.656 to 51.442 & 4.72 to 51.424 \\
\hline Index ranges & $\begin{array}{c}-14 \leq \mathrm{h} \leq 14 \\
-16 \leq \mathrm{k} \leq 17 \\
-18 \leq \mathrm{I} \leq 18\end{array}$ & $\begin{array}{r}-22 \leq \mathrm{h} \leq 22 \\
-53 \leq \mathrm{k} \leq 53 \\
-22 \leq \mathrm{I} \leq 22\end{array}$ & $\begin{array}{c}-23 \leq h \leq 23 \\
-24 \leq k \leq 24 \\
-24 \leq 1 \leq 24\end{array}$ & $\begin{array}{c}-23 \leq h \leq 28 \\
-24 \leq k \leq 24 \\
-31 \leq 1 \leq 31\end{array}$ \\
\hline Total reflns & 215440 & 586520 & 100247 & 100523 \\
\hline Ind. reflns / R(int) & $9603 / 0.0391$ & $28711 / 0.1101$ & $22550 / 0.1741$ & 11717 / 0.0511 \\
\hline $\begin{array}{c}\text { Completeness, } \\
\text { Omax \% } \\
\end{array}$ & 99.0 & 99.6 & 99.4 & 99.4 \\
\hline Goof( F2) & 1.097 & 1.017 & 0.838 & 1.158 \\
\hline $\mathrm{R} 1 / \mathrm{wR}_{2}(\mathrm{~F})[\mathrm{I}>2 \sigma(\mathrm{I})]$ & $0.0176 / 0.0409$ & $0.0342 / 0.0689$ & $0.0727 / 0.1901$ & $0.0716 / 0.2180$ \\
\hline $\mathrm{wR}_{2}\left(\mathrm{~F}^{2}\right)$ (all data) & 0.0419 & 0.0742 & 0.2371 & 0.2341 \\
\hline $\begin{array}{c}\text { Largest diff } \\
\text { peak/hole, e. } \AA \text { - } 3\end{array}$ & $0.56 /-0.71$ & $1.80 /-1.54$ & $0.77 /-0.40$ & $1.07 /-0.56$ \\
\hline
\end{tabular}




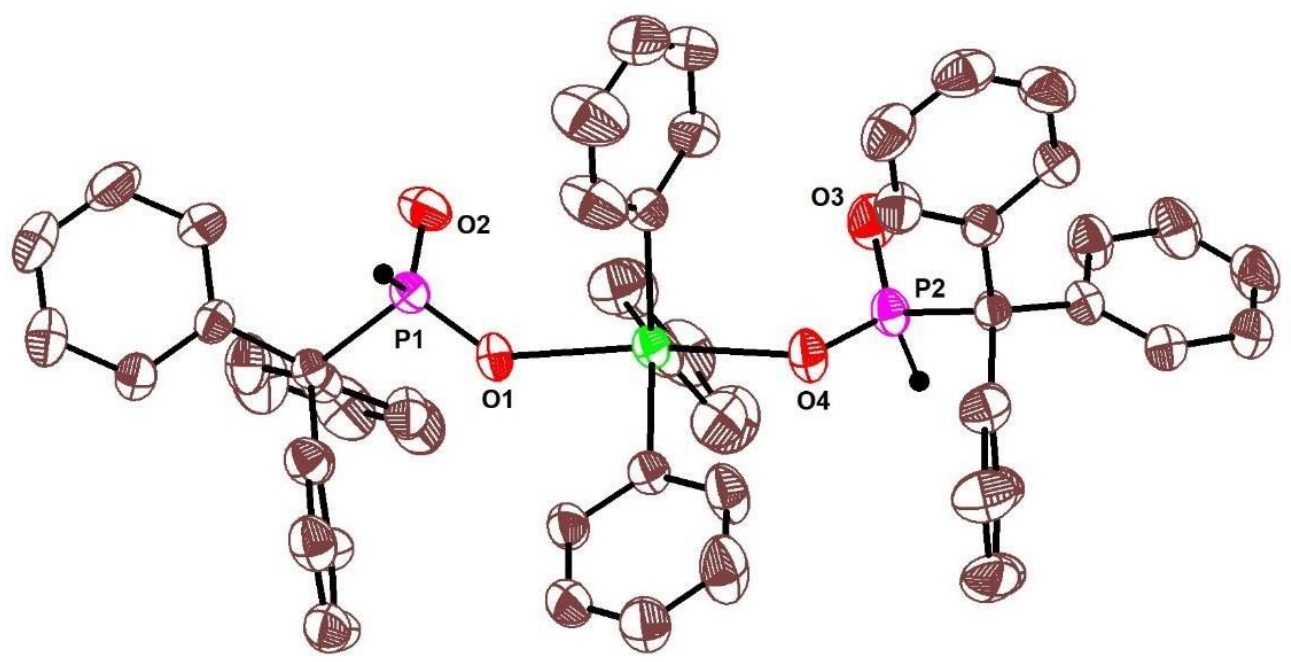

Figure S1. ORTEP view of $\mathbf{1}$ with thermal ellipsoids shown at 30\% probability.

Table S2: Selected bond lengths $(\AA)$ and bond angle (deg) parameters of compound $\mathbf{1}$.

\begin{tabular}{|ll|ll|ll|}
\hline $\mathrm{Bi}(1)-\mathrm{O}(1)$ & $2.239(15)$ & $\mathrm{Bi}(1)-\mathrm{O}(4)$ & $2.234(14)$ & $\mathrm{O}(4)-\mathrm{Bi}(1)-\mathrm{O}(4)$ & $172.91(6)$ \\
\hline
\end{tabular}

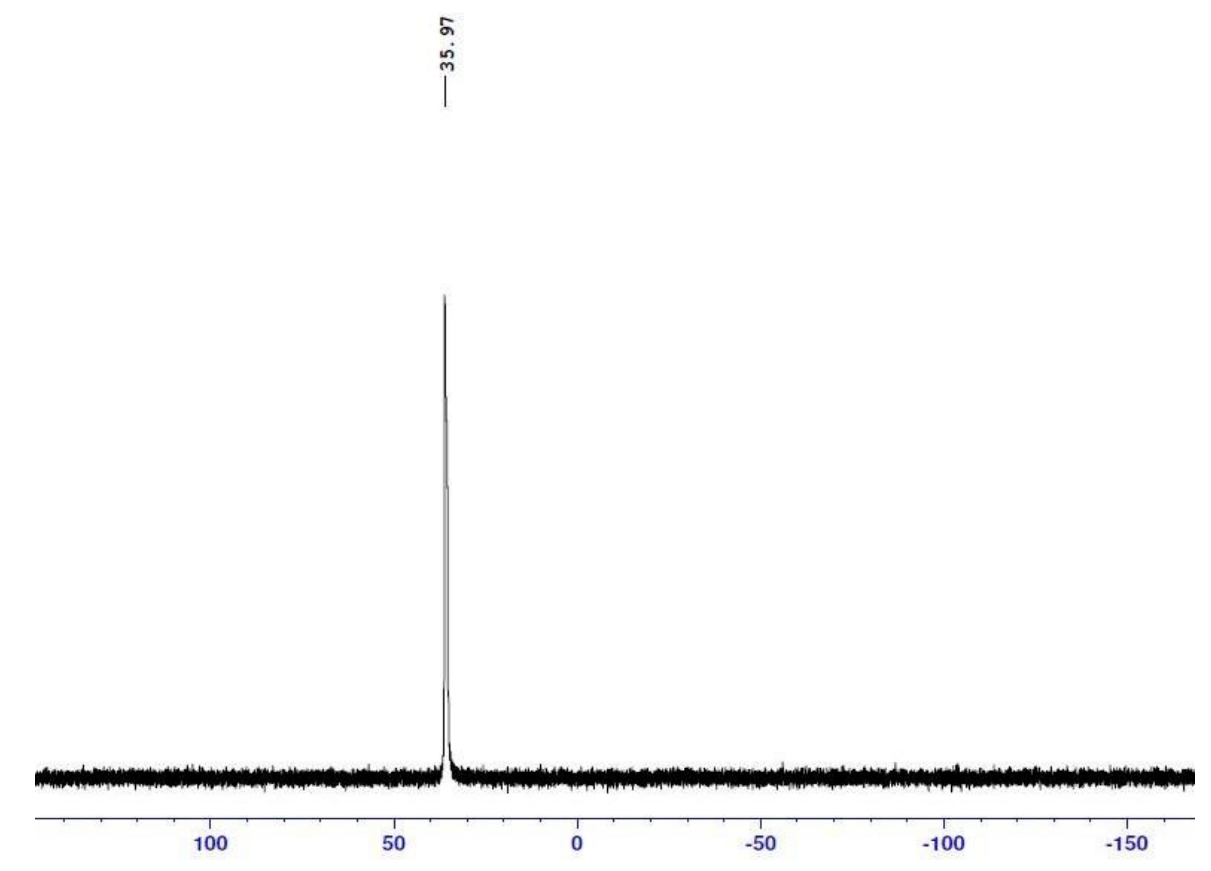

Figure S2: ${ }^{31} \mathbf{P}$ NMR $\left(162 \mathrm{MHz}, \mathrm{CDCl}_{3}\right)$ of compound 1. 


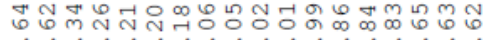

-

shamath ald

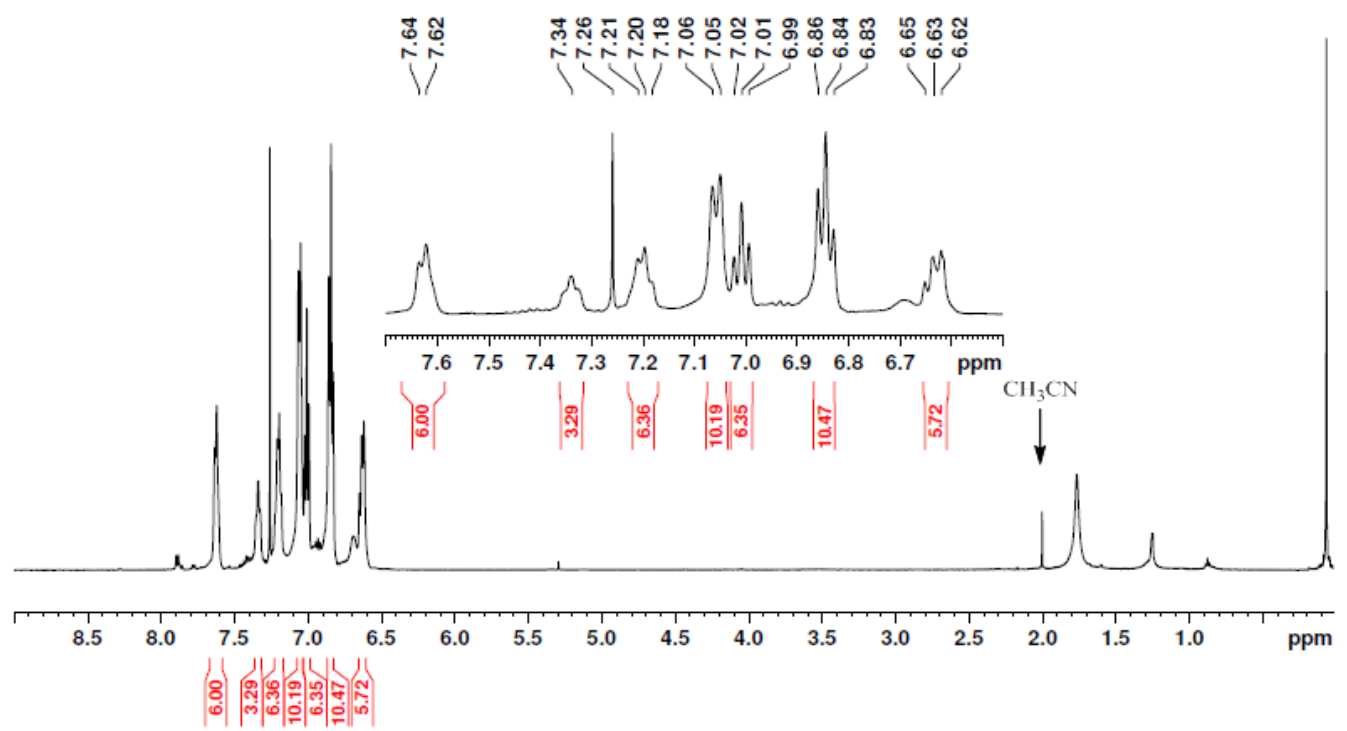

Figure S3: ${ }^{1} \mathrm{H}$ NMR $\left(500 \mathrm{MHz}, \mathrm{CDCl}_{3}\right)$ of compound 1.

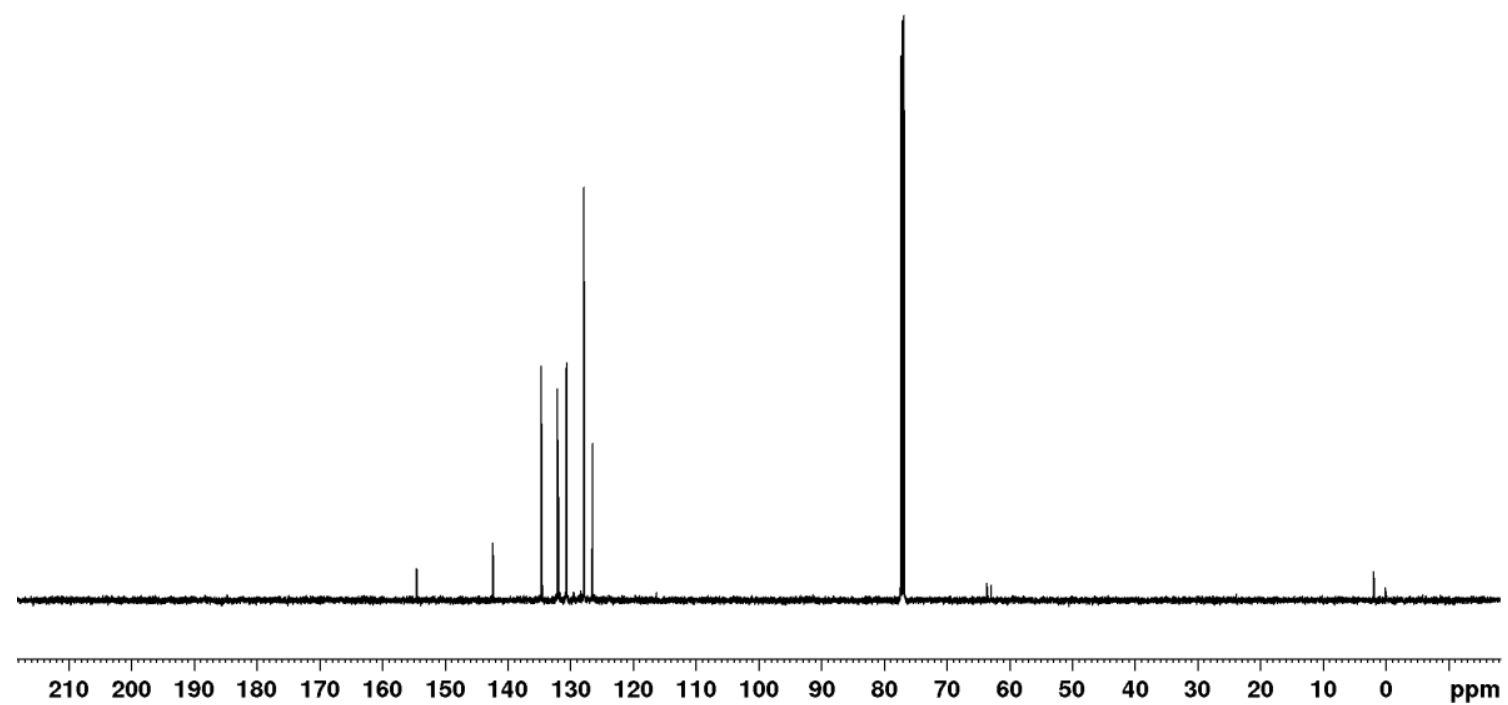

Figure S4: ${ }^{13} \mathrm{C}$ NMR (100 MHz, $\left.\mathrm{CDCl}_{3}\right)$ of compound $\mathbf{1}$. 


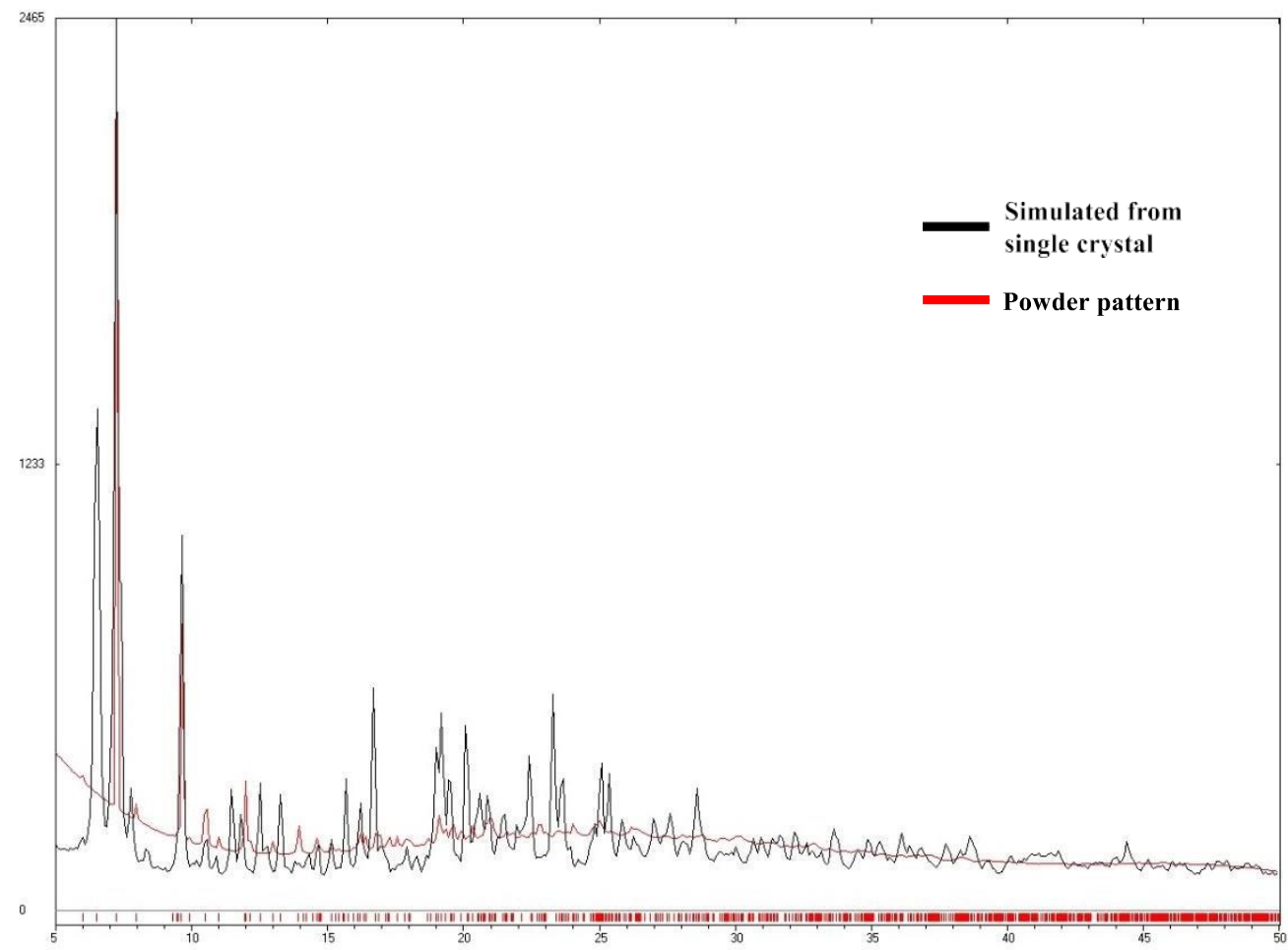

Figure S5: Powder X-ray diffraction pattern of a bulk sample of $\mathbf{1}$ compared to the simulated powder pattern extracted from single crystal diffraction data.

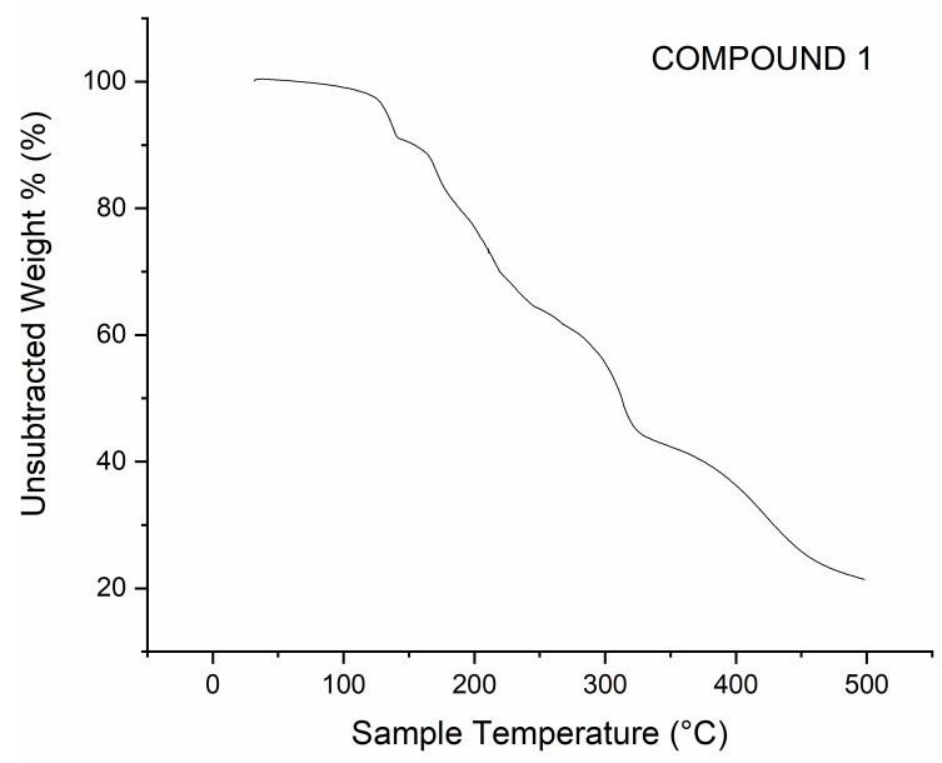

Figure S6: The TGA curve of 1 heating to $500{ }^{\circ} \mathrm{C}$ in $\mathrm{N}_{2}$ at a rate of $10{ }^{\circ} \mathrm{C}$ min. 


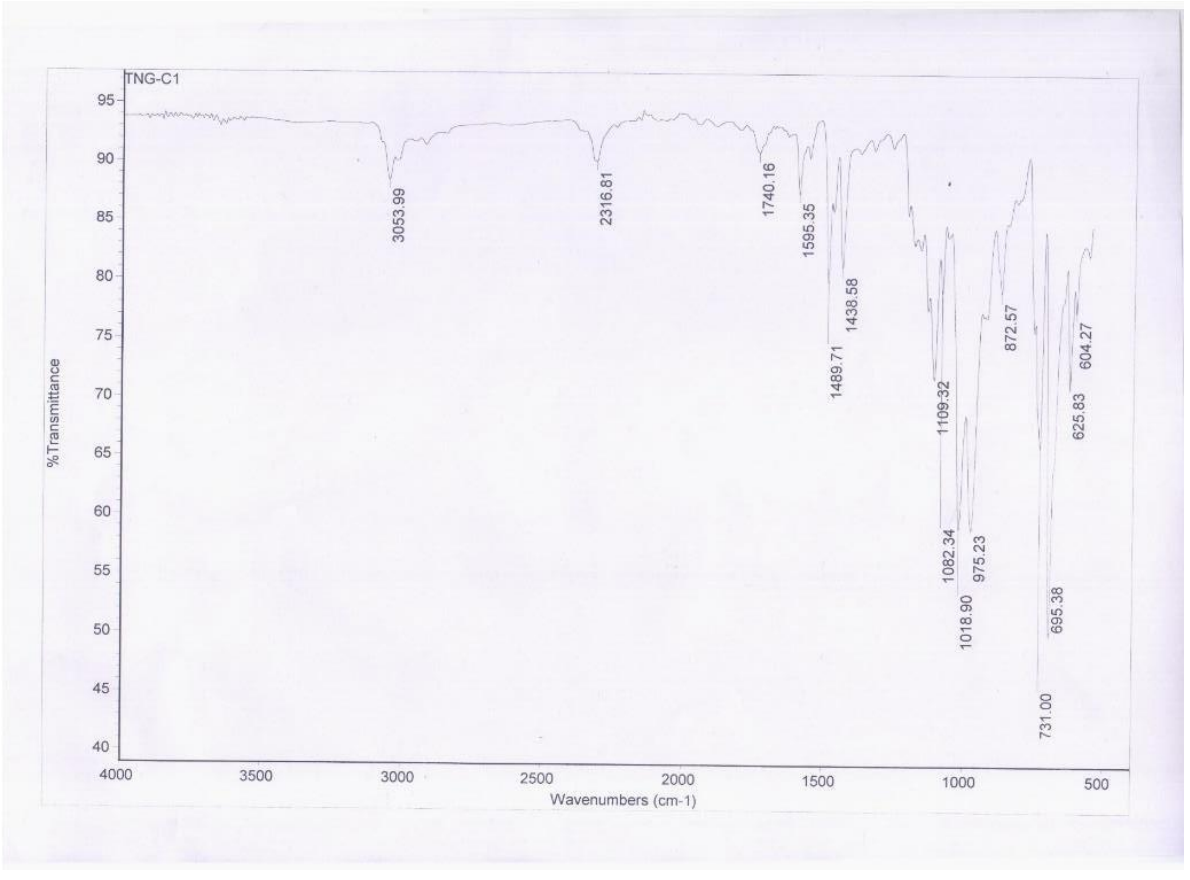

Figure S7: IR spectra of compound $1.3055 \mathrm{~cm}^{-1}$ (aromatic C-H stretching), $2316 \mathrm{~cm}^{-1}(\mathrm{P}-\mathrm{H}$ stretching), $1109 \mathrm{~cm}^{-1}, 1082 \mathrm{~cm}^{-1}, 1018 \mathrm{~cm}^{-1}$ (P-O-M stretching).
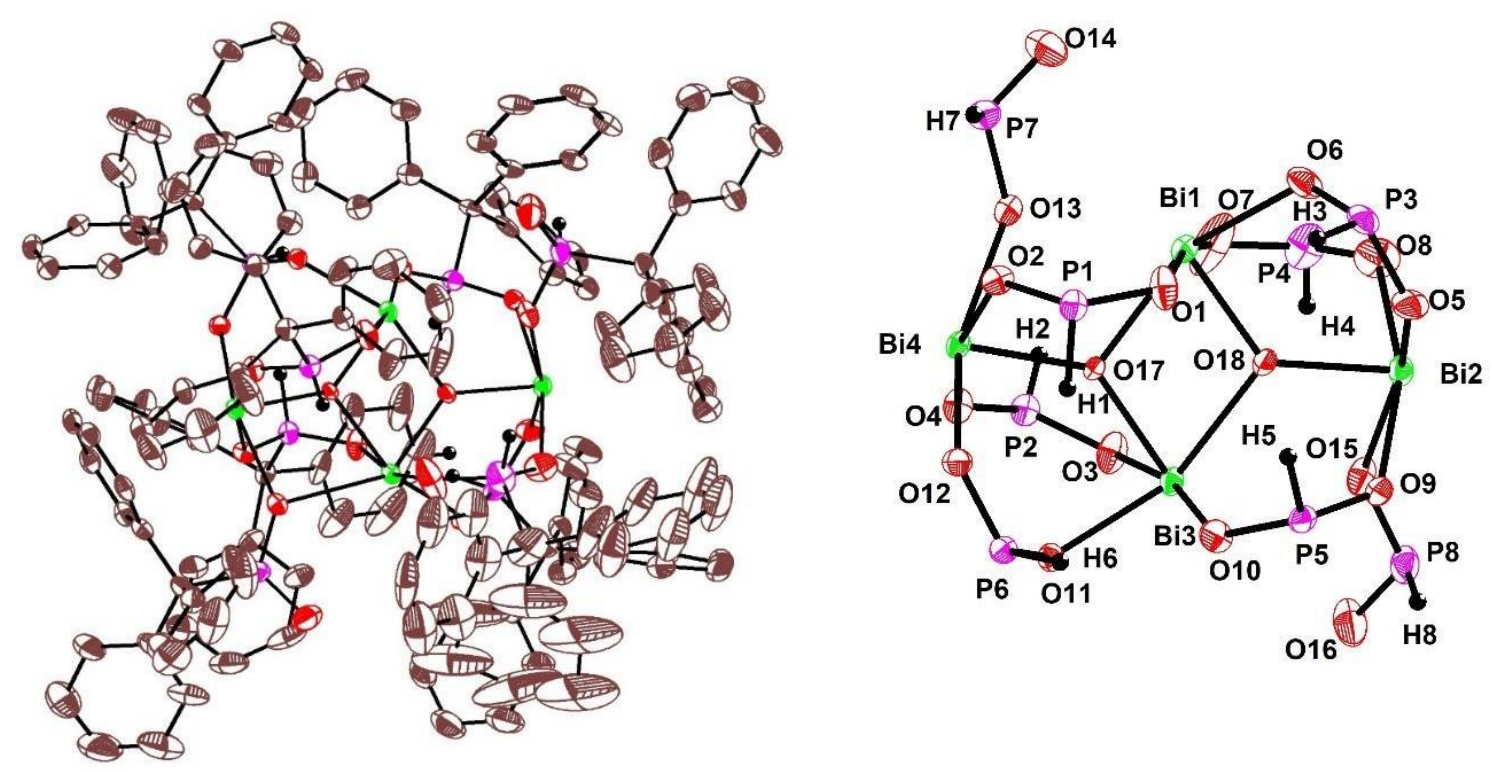

Figure S8. ORTEP view of $\mathbf{2}$ and its labelled metal oxo core with thermal ellipsoids shown at 30\% probability. 
Table S3: Selected bond lengths $(\AA)$ and bond angles (deg) parameters of compound 2.

\begin{tabular}{|c|c|c|c|}
\hline $\mathrm{Bi}(2)-\mathrm{O}(18)$ & $2.096(3)$ & $\mathrm{Bi}(4)-\mathrm{O}(4)$ & $\mathrm{O}(1)-\mathrm{Bi}(1)-\mathrm{O}(19) \quad 90.24(13)$ \\
\hline $\mathrm{Bi}(2)-\mathrm{O}(15)$ & $2.287(3)$ & $\mathrm{O}(18)-\mathrm{Bi}(2)-\mathrm{O}(15) 78.82(11)$ & $\mathrm{O}(7)-\mathrm{Bi}(1)-\mathrm{Bi}(3)$ \\
\hline $\mathrm{Bi}(2)-\mathrm{O}(8)$ & $2.339(3)$ & $\mathrm{O}(18)-\mathrm{Bi}(2)-\mathrm{O}(8) \quad 82.71(11)$ & $\mathrm{O}(7)-\mathrm{Bi}(1)-\mathrm{O}(17)$ 111.73(12) \\
\hline $\mathrm{Bi}(2)-\mathrm{O}(5)$ & $2.318(3)$ & $\mathrm{O}(18)-\mathrm{Bi}(2)-\mathrm{O}(5) \quad 85.22(11)$ & $\mathrm{O}(7)-\mathrm{Bi}(1)-\mathrm{O}(19) \quad 81.16(13)$ \\
\hline $\mathrm{Bi}(2)-\mathrm{O}(9)$ & $2.303(3)$ & $\mathrm{O}(18)-\mathrm{Bi}(2)-\mathrm{O}(9) \quad 82.74(11)$ & $\mathrm{O}(18)-\mathrm{Bi}(3)-\mathrm{O}(11)$ 148.7(11) \\
\hline $\mathrm{Bi}(1)-\mathrm{O}(18)$ & $2.171(3)$ & $\mathrm{O}(15)-\mathrm{Bi}(2)-\mathrm{O}(8) \quad 93.97(12)$ & $\mathrm{O}(18)-\mathrm{Bi}(3)-\mathrm{O}(10) 80.15(12)$ \\
\hline $\mathrm{Bi}(1)-\mathrm{O}(17)$ & $2.412(3)$ & $\mathrm{O}(15)-\mathrm{Bi}(2)-\mathrm{O}(9) \quad 78.68(12)$ & $\mathrm{O}(18)-\mathrm{Bi}(3)-\mathrm{O}(3) \quad 90.77(16)$ \\
\hline $\mathrm{Bi}(1)-\mathrm{O}(6)$ & $2.278(3)$ & $\mathrm{O}(5)-\mathrm{Bi}(2)-\mathrm{O}(8) \quad 106.35(11)$ & $\mathrm{O}(17)-\mathrm{Bi}(3)-\mathrm{O}(18) 72.06(11)$ \\
\hline $\mathrm{Bi}(1)-\mathrm{O}(1)$ & $2.360(3)$ & $\mathrm{O}(9)-\mathrm{Bi}(2)-\mathrm{O}(5)$ & $\mathrm{O}(17)-\mathrm{Bi}(3)-\mathrm{O}(11) 86.08(12)$ \\
\hline $\mathrm{Bi}(1)-\mathrm{O}(7)$ & $2.367(3)$ & $\mathrm{O}(18)-\mathrm{Bi}(1)-\mathrm{Bi}(3) \quad 36.25(8)$ & $\mathrm{O}(17)-\mathrm{Bi}(3)-\mathrm{O}(10) 86.22(13)$ \\
\hline $\mathrm{Bi}(1)-\mathrm{O}(19)$ & $2.674(4)$ & $\mathrm{O}(18)-\mathrm{Bi}(1)-\mathrm{O}(17)$ 68.52(10) & $\mathrm{O}(17)-\mathrm{Bi}(3)-\mathrm{O}(3) \quad 81.89(13)$ \\
\hline $\mathrm{Bi}(3)-\mathrm{O}(17)$ & $2.108(3)$ & $\mathrm{O}(18)-\mathrm{Bi}(1)-\mathrm{O}(6) \quad 82.74(11)$ & $\mathrm{O}(11)-\mathrm{Bi}(3)-\mathrm{O}(3) 108.29(16)$ \\
\hline $\mathrm{Bi}(3)-\mathrm{O}(11)$ & $2.355(3)$ & $\mathrm{O}(18)-\mathrm{Bi}(1)-\mathrm{O}(1) \quad 99.99(12)$ & $\mathrm{O}(10)-\mathrm{Bi}(3)-\mathrm{O}(11) 76.35(12)$ \\
\hline $\mathrm{Bi}(3)-\mathrm{O}(10)$ & $2.313(3)$ & $\mathrm{O}(18)-\mathrm{Bi}(1)-\mathrm{O}(7) \quad 84.88(12)$ & $\mathrm{O}(17)-\mathrm{Bi}(4)-\mathrm{O}(2) \quad 82.23(12)$ \\
\hline $\mathrm{Bi}(3)-\mathrm{O}(3)$ & $2.388(4)$ & $\mathrm{O}(17)-\mathrm{Bi}(1)-\mathrm{O} 19$ 138.37(11) & $\mathrm{O}(17)-\mathrm{Bi}(4)-\mathrm{O}(13) 80.22(12)$ \\
\hline $\mathrm{Bi}(3)-\mathrm{O} 18$ & $2.285(3)$ & $\mathrm{O}(6)-\mathrm{Bi}(1)-\mathrm{O}(17) 138.80(11)$ & $\mathrm{O}(17)-\mathrm{Bi}(4)-\mathrm{O}(12) 88.41(12)$ \\
\hline $\mathrm{Bi}(4)-\mathrm{O}(17)$ & $2.074(3)$ & $\mathrm{O}(6)-\mathrm{Bi}(1)-\mathrm{O}(1)$ & $\mathrm{O}(17)-\mathrm{Bi}(4)-\mathrm{O}(4)$ \\
\hline $\mathrm{Bi}(4)-\mathrm{O}(2)$ & $2.261(3)$ & $\mathrm{O}(6)-\mathrm{Bi}(1)-\mathrm{O}(7)$ & $\mathrm{O}(2)-\mathrm{Bi}(4)-\mathrm{O}(13)$ \\
\hline $\mathrm{Bi}(4)-\mathrm{O}(13)$ & $2.330(3)$ & $\mathrm{O}(6)-\mathrm{Bi}(1)-\mathrm{O}(19) \quad 75.19(12)$ & $\mathrm{O}(2)-\mathrm{Bi}(4)-\mathrm{O}(12)$ \\
\hline $\mathrm{Bi}(4)-\mathrm{O}(12)$ & $2.324(3)$ & $\mathrm{O}(1)-\mathrm{Bi}(1)-\mathrm{O}(17)$ & $\mathrm{O}(13)-\mathrm{Bi}(4)-\mathrm{O}(4)$ \\
\hline
\end{tabular}




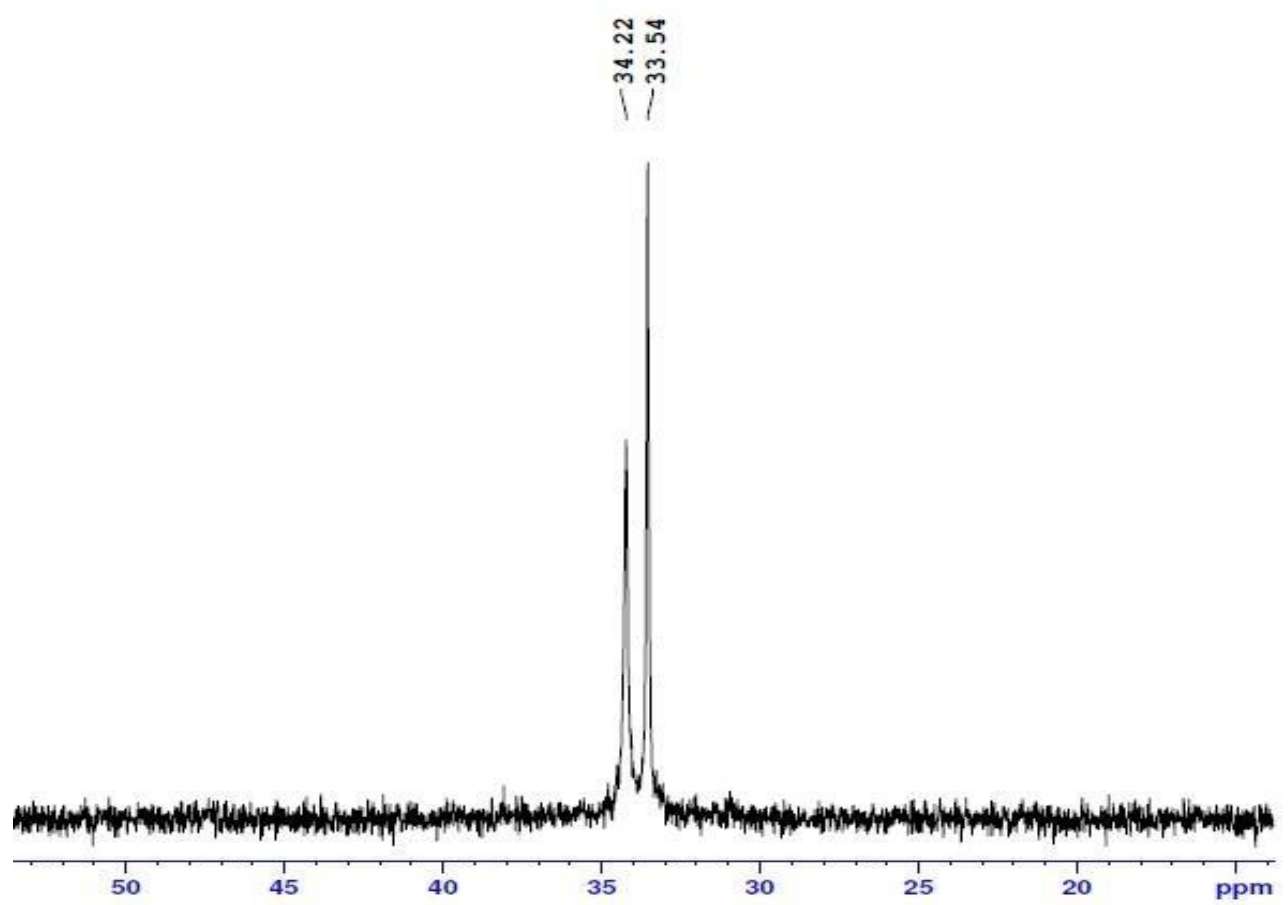

Figure S9: ${ }^{31} \mathbf{P}$ NMR (162 MHz, $\left.\mathrm{CDCl}_{3}\right)$ of compound 2.

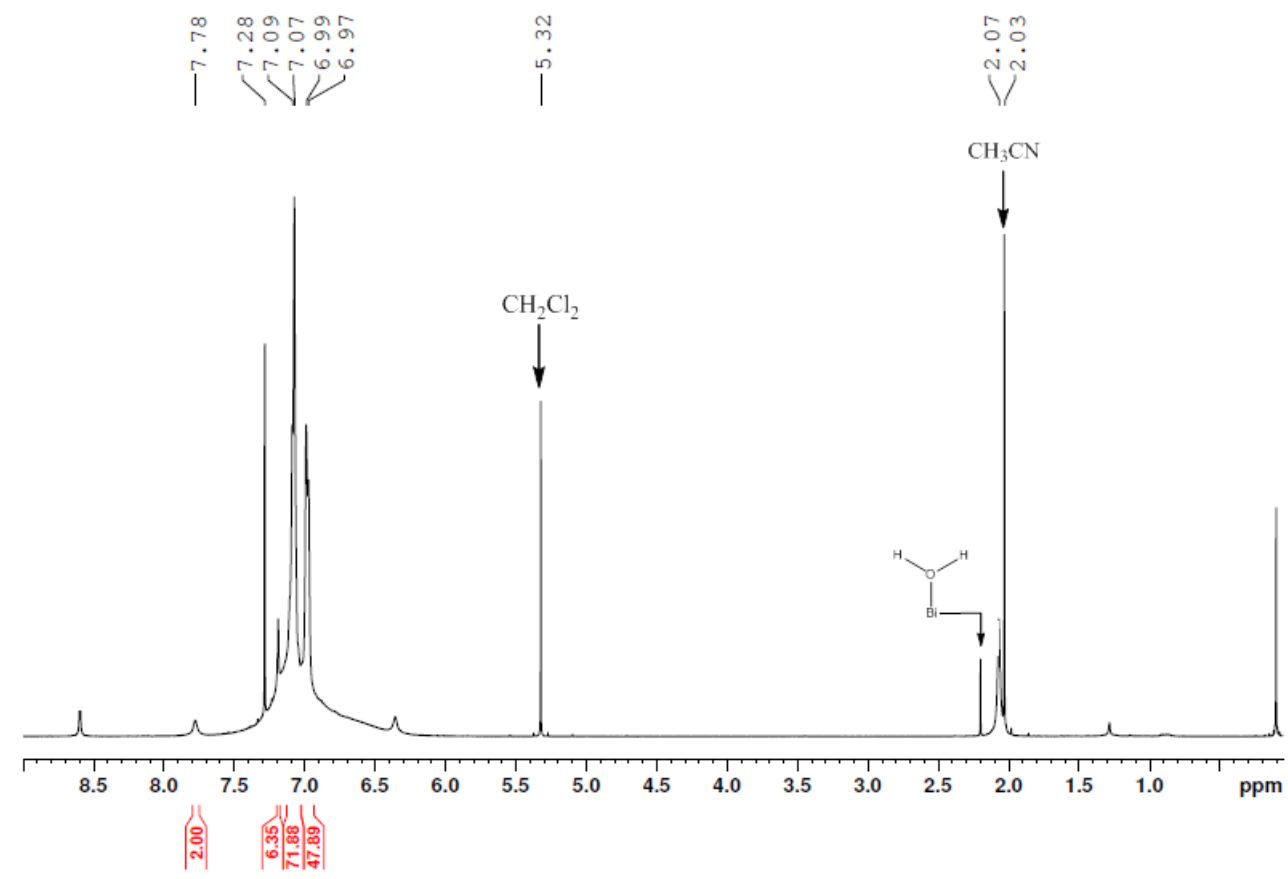

Figure S10: ${ }^{1} \mathbf{H} \mathrm{NMR}\left(500 \mathrm{MHz}, \mathrm{CDCl}_{3}\right)$ of compound 2. 


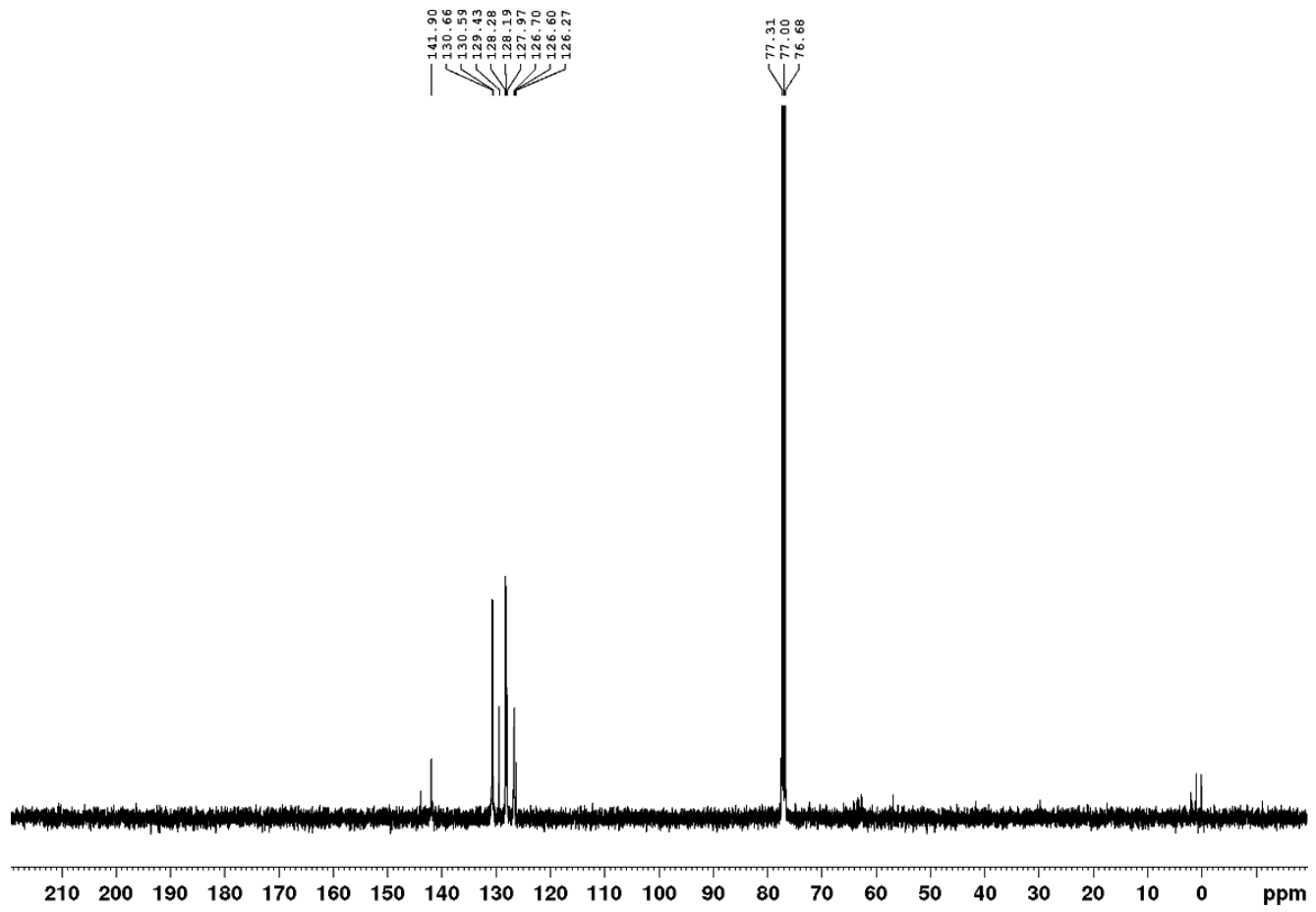

Figure S11: ${ }^{13} \mathrm{C}$ NMR $\left(100 \mathrm{MHz}, \mathrm{CDCl}_{3}\right)$ of compound 2.

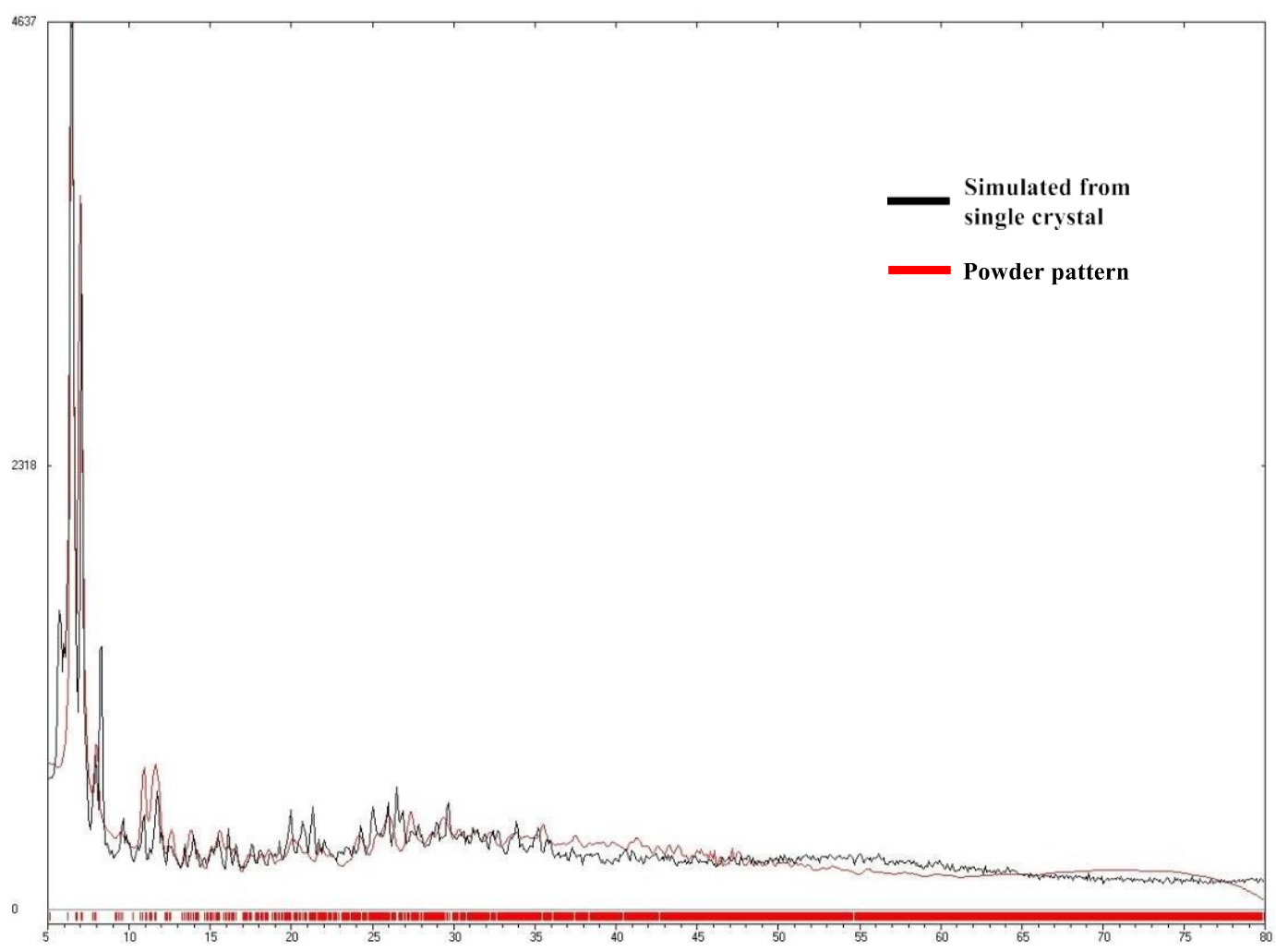

Figure S12: Powder X-ray diffraction pattern of a bulk sample of $\mathbf{2}$ compared to the simulated powder pattern extracted from single crystal diffraction data. 


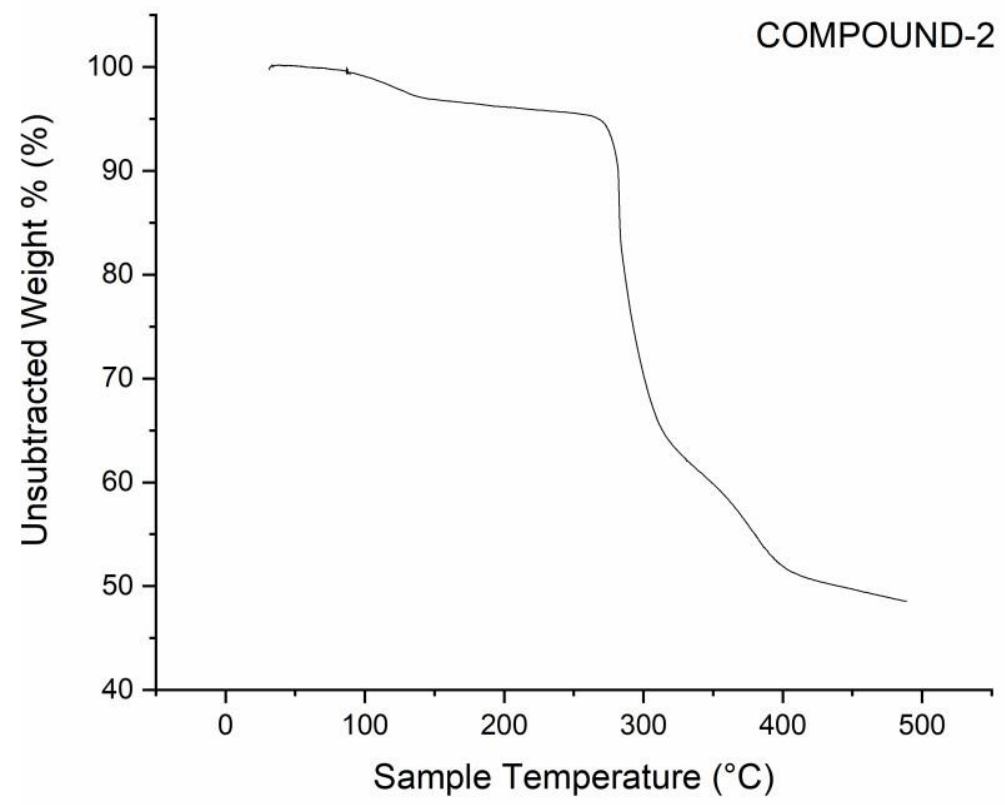

Figure S13: The TGA curve of 2 heating to $500{ }^{\circ} \mathrm{C}$ in $\mathrm{N}_{2}$ at a rate of $10{ }^{\circ} \mathrm{C}$ min.

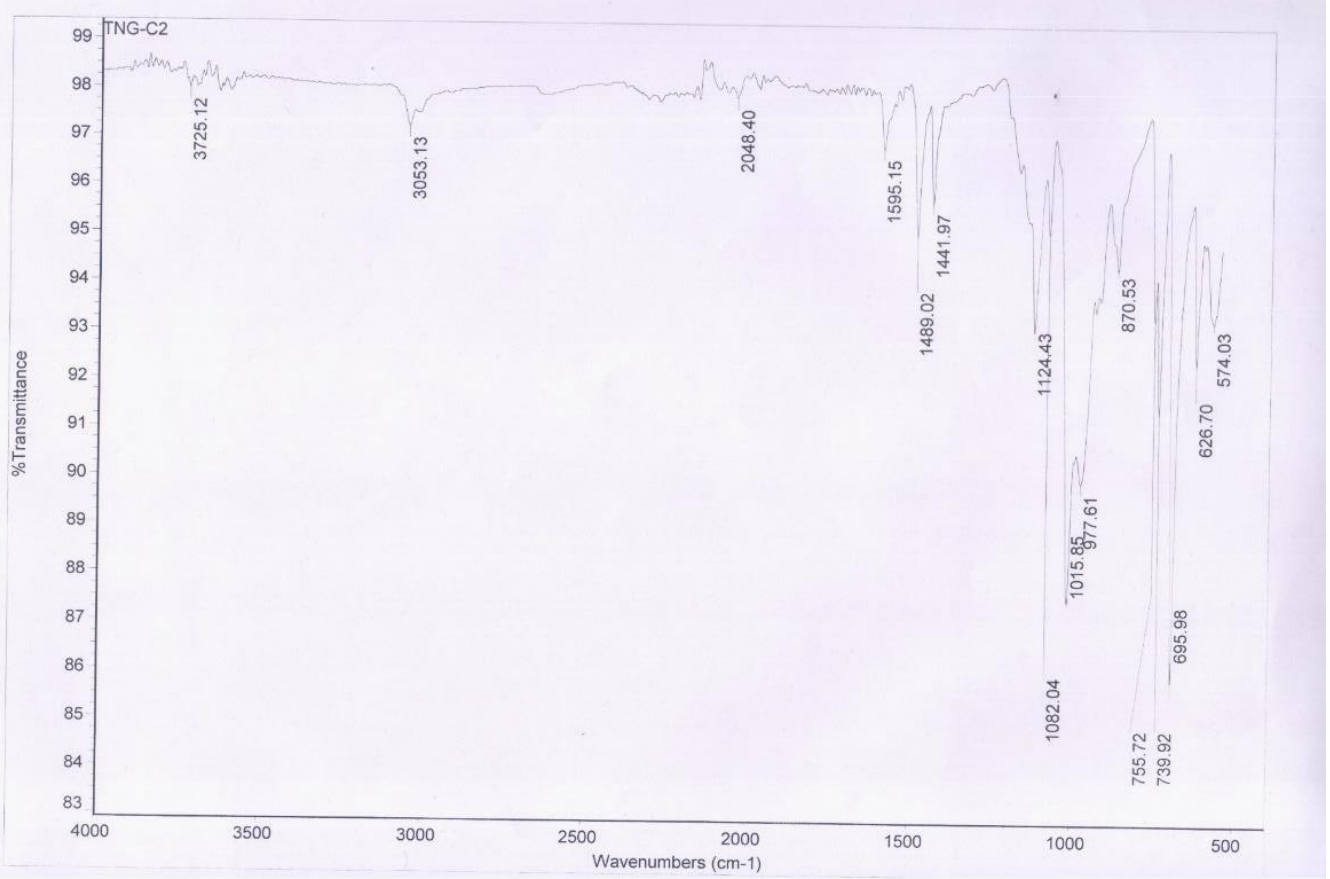

Figure S14: IR spectra of compound 2. $2955 \mathrm{~cm}^{-1}$ (aromatic C-H stretching), $2359 \mathrm{~cm}^{-1}(\mathrm{P}-\mathrm{H}$ stretching), 1259, 1236 ( $\mathrm{P}=\mathrm{O}$ stretching) $1080 \mathrm{~cm}^{-1}, 1000 \mathrm{~cm}^{-1}$ (P-O-M stretching). 

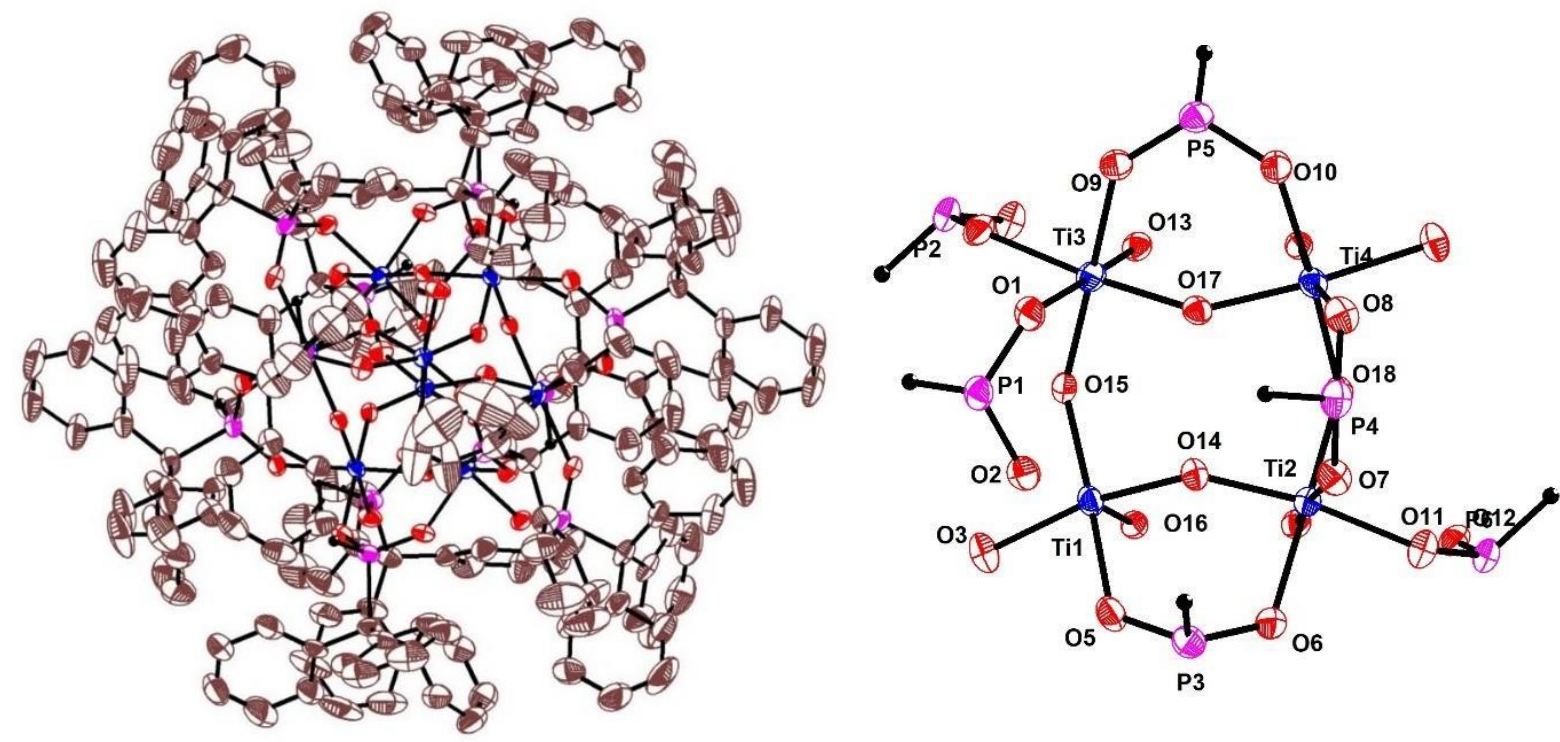

Figure S15. ORTEP view of $\mathbf{3}$ and its asymmetric unit with thermal ellipsoids shown at $30 \%$ probability.

Table S4: Selected bond lengths ( $\mathrm{A})$ and bond angles (deg) parameters of compound 3.

\begin{tabular}{|c|c|c|c|}
\hline $\mathrm{Ti}(1)-\mathrm{O}(1)$ & $1.999(3)$ & $\mathrm{Ti}(4)-\mathrm{O}(18)$ & $\mathrm{O}(21)-\mathrm{Ti}(3)-\mathrm{O}(12) 90.74(13)$ \\
\hline $\mathrm{Ti}(1)-\mathrm{O}(3)$ & $2.042(3)$ & $\mathrm{O}(1)-\mathrm{Ti}(1)-\mathrm{O}(3) \quad 87.31(13)$ & $\mathrm{O}(9)-\mathrm{Ti}(3)-\mathrm{O}(2)^{1} \quad 88.94(12)$ \\
\hline $\mathrm{Ti}(1)-\mathrm{O}(5)$ & $2.028(3)$ & $\mathrm{O}(1)-\mathrm{Ti}(1)-\mathrm{O}(5)$ & $\mathrm{O}(9)-\mathrm{Ti}(3)-\mathrm{O}(12) \quad 91.16(13)$ \\
\hline $\mathrm{Ti}(1)-\mathrm{O}(14)$ & $1.843(3)$ & $\mathrm{O}(5)-\mathrm{Ti}(1)-\mathrm{O}(3)$ & $\mathrm{O}(13)-\mathrm{Ti}(3)-\mathrm{O}(9) \quad 94.38(13)$ \\
\hline $\mathrm{Ti}(1)-\mathrm{O}(15)$ & $1.922(3)$ & $\mathrm{O}(14)-\mathrm{Ti}(1)-\mathrm{O}(1) \quad 97.70(13)$ & $\mathrm{O}(13)-\mathrm{Ti}(3)-\mathrm{O}(12) 85.00(13)$ \\
\hline $\mathrm{Ti}(1)-\mathrm{O}(16)$ & $1.912(3)$ & $\mathrm{O}(14)-\mathrm{Ti}(1)-\mathrm{O}(5) \quad 84.26(14)$ & $\mathrm{O}(13)-\mathrm{Ti}(3)-\mathrm{O}(15) 92.61(13)$ \\
\hline $\mathrm{Ti}(2)-\mathrm{O}(6)$ & $2.087(3)$ & $\mathrm{O}(14)-\mathrm{Ti}(1)-\mathrm{O}(15)$ 94.13(13) & $\mathrm{O}(13)-\mathrm{Ti}(3)-\mathrm{O}(17) 91.84(13)$ \\
\hline $\mathrm{Ti}(2)-\mathrm{O}(7)$ & $2.046(3)$ & $\mathrm{O}(14)-\mathrm{Ti}(1)-\mathrm{O}(16) 92.73(13)$ & $\mathrm{O}(15)^{1}-\mathrm{Ti}(3)-\mathrm{O}(2)^{1} 84.21(12)$ \\
\hline $\mathrm{Ti}(2)-\mathrm{O}(11)$ & $1.997(3)$ & $\mathrm{O}(15)-\mathrm{Ti}(1)-\mathrm{O}(1)$ & $\mathrm{O}(15)^{1}-\mathrm{Ti}(3)-\mathrm{O}(12) 91.04(13)$ \\
\hline $\mathrm{Ti}(2)-\mathrm{O}(13)$ & $1.880(3)$ & $\mathrm{O}(15)-\mathrm{Ti}(1)-\mathrm{O}(3)$ & $\mathrm{O}(17)-\mathrm{Ti}(3)-\mathrm{O}(2)^{1} \quad 92.62(13)$ \\
\hline $\mathrm{Ti}(2)-\mathrm{O}(14)$ & $1.878(3)$ & $\mathrm{O}(16)-\mathrm{Ti}(1)-\mathrm{O}(3)$ & $\mathrm{O}(17)-\mathrm{Ti}(3)-\mathrm{O}(9)$ \\
\hline
\end{tabular}




\begin{tabular}{|c|c|c|c|}
\hline $\mathrm{Ti}(2)-\mathrm{O}(18)$ & $1.868(3)$ & $\mathrm{O}(16)-\mathrm{Ti}(1)-\mathrm{O}(5) \quad 99.04(13)$ & $\mathrm{O}(17)-\mathrm{Ti}(3)-\mathrm{O}(15) 92.84(13)$ \\
\hline $\mathrm{Ti}(3)-\mathrm{O}(2)^{1}$ & $2.083(3)$ & $\mathrm{O}(16)-\mathrm{Ti}(1)-\mathrm{O}(15)$ 92.12(13) & $\mathrm{O}(8)-\mathrm{Ti}(4)-\mathrm{O}(4)^{1} \quad 88.80(13)$ \\
\hline $\mathrm{Ti}(3)-\mathrm{O}(9)$ & $2.024(3)$ & $\mathrm{O}(7)-\mathrm{Ti}(2)-\mathrm{O}(6)$ & $\mathrm{O}(8)-\mathrm{Ti}(4)-\mathrm{O}(10)^{1} \quad 88.72(13)$ \\
\hline $\mathrm{Ti}(3)-\mathrm{O}(12)$ & $2.093(3)$ & $\mathrm{O}(11)-\mathrm{Ti}(2)-\mathrm{O}(6) \quad 85.87(13)$ & $\mathrm{O}(10)-\mathrm{Ti}(4)-\mathrm{O}(4)^{1} \quad 91.67(13)$ \\
\hline $\mathrm{Ti}(3)-\mathrm{O}(13)$ & $1.864(3)$ & $\mathrm{O}(11)-\mathrm{Ti}(2)-\mathrm{O}(7) \quad 88.12(13)$ & $\mathrm{O}(16)^{1}-\mathrm{Ti}(4)-\mathrm{O}(4)^{1} 84.49(13)$ \\
\hline $\mathrm{Ti}(3)-\mathrm{O}(15)^{1}$ & $1.923(3)$ & $\mathrm{O}(13)-\mathrm{Ti}(2)-\mathrm{O}(6) \quad 92.32(13)$ & $\mathrm{O}(16)^{1}-\mathrm{Ti}(4)-\mathrm{O}(10) 90.25(13)$ \\
\hline $\mathrm{Ti}(3)-\mathrm{O}(17)$ & $1.899(3)$ & $\mathrm{O}(13)-\mathrm{Ti}(2)-\mathrm{O}(11) 85.88(13)$ & $\mathrm{O}(16)^{1}-\mathrm{Ti}(4)-\mathrm{O}(17)^{1} 92.7(13)$ \\
\hline $\operatorname{Ti}(4)-O(4)^{1}$ & $2.060(3)$ & $\mathrm{O}(14)-\mathrm{Ti}(2)-\mathrm{O}(6)$ & $\mathrm{O}(17)^{1}-\mathrm{Ti}(4)-\mathrm{O}(8) \quad 93.83(13)$ \\
\hline $\mathrm{Ti}(4)-\mathrm{O}(8)$ & $2.025(3)$ & $\mathrm{O}(14)-\mathrm{Ti}(2)-\mathrm{O}(7) \quad 91.69(13)$ & $\mathrm{O}(17)^{1}-\mathrm{Ti}(4)-\mathrm{O} 10^{1} 84.05(12)$ \\
\hline $\mathrm{Ti}(4)-\mathrm{O}(10)^{1}$ & $2.055(3)$ & $\mathrm{O}(14)-\mathrm{Ti}(2)-\mathrm{O}(13) 94.67(13)$ & $\mathrm{O}(18)-\mathrm{Ti}(4)-\mathrm{O}(4)^{1} 92.49(13)$ \\
\hline $\mathrm{Ti}(4)-\mathrm{O}(16)^{1}$ & $1.913(3)$ & $\mathrm{O}(18)-\mathrm{Ti}(2)-\mathrm{O}(7)$ & $\mathrm{O}(18)-\mathrm{Ti}(4)-\mathrm{O}(8) \quad 87.51(13)$ \\
\hline $\mathrm{Ti}(4)-\mathrm{O}(17)^{1}$ & $1.917(3)$ & $\mathrm{O}(18)-\mathrm{Ti}(2)-\mathrm{O}(14) 93.87(13)$ & $\mathrm{O}(18)-\mathrm{Ti}(4)-\mathrm{O}(16)^{1} 93.9(12)$ \\
\hline
\end{tabular}

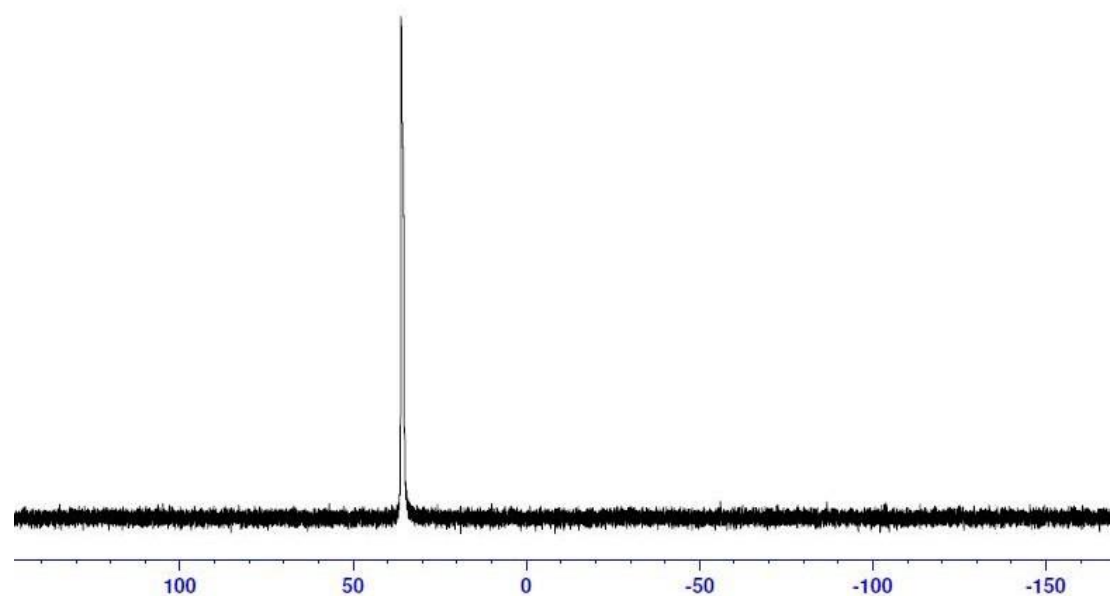

Figure S16: ${ }^{31} \mathrm{P}$ NMR $\left(162 \mathrm{MHz}, \mathrm{CDCl}_{3}\right)$ of compound 3. 


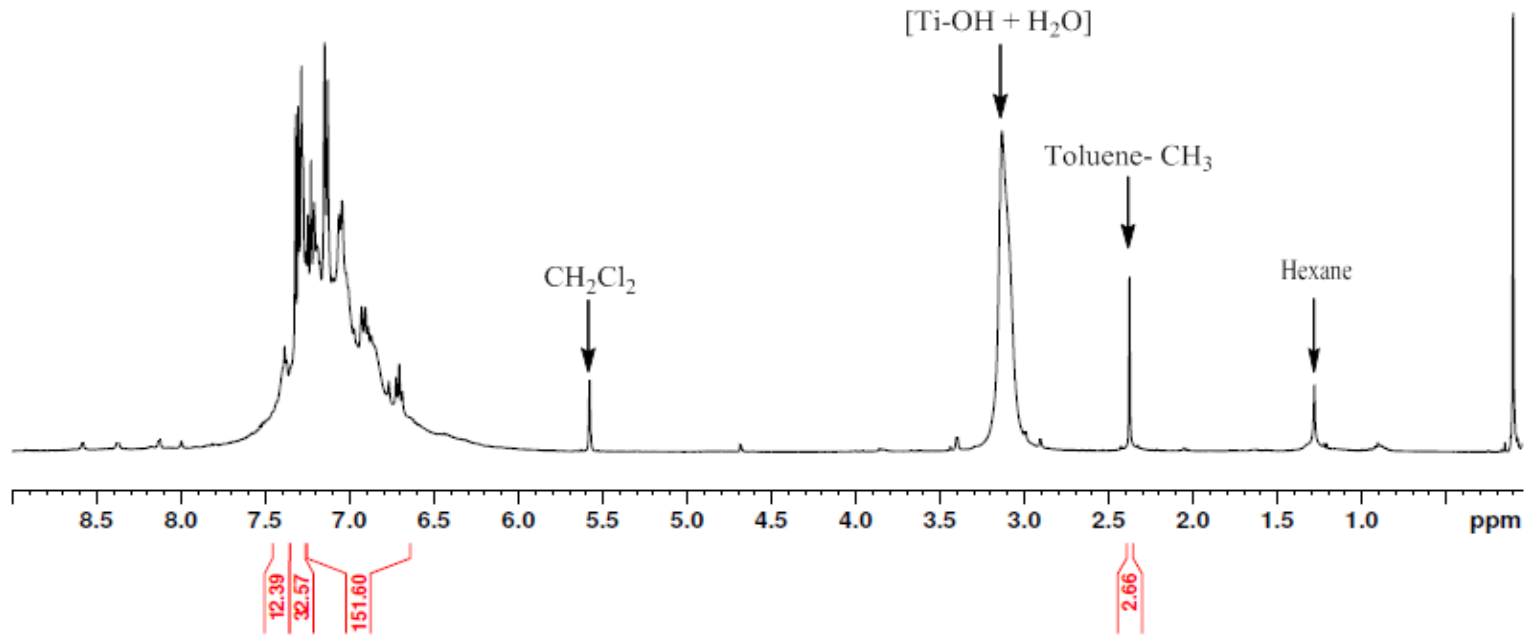

Figure S17: ${ }^{1} \mathbf{H}$ NMR $\left(500 \mathrm{MHz}, \mathrm{CDCl}_{3}\right)$ of compound 3.

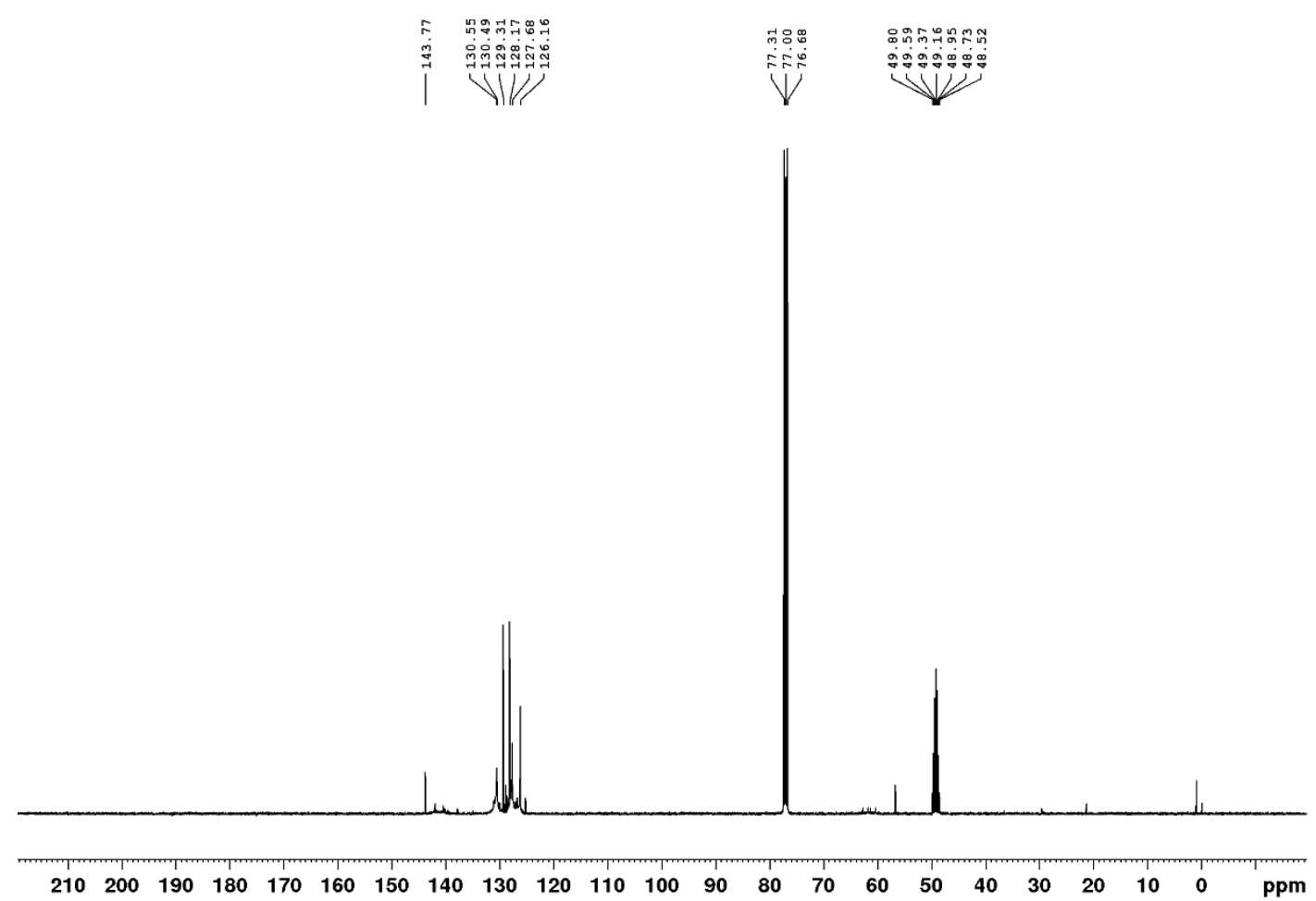

Figure S18: ${ }^{13} \mathrm{C}$ NMR $\left(100 \mathrm{MHz}, \mathrm{CDCl}_{3}\right)$ of compound 3 . 


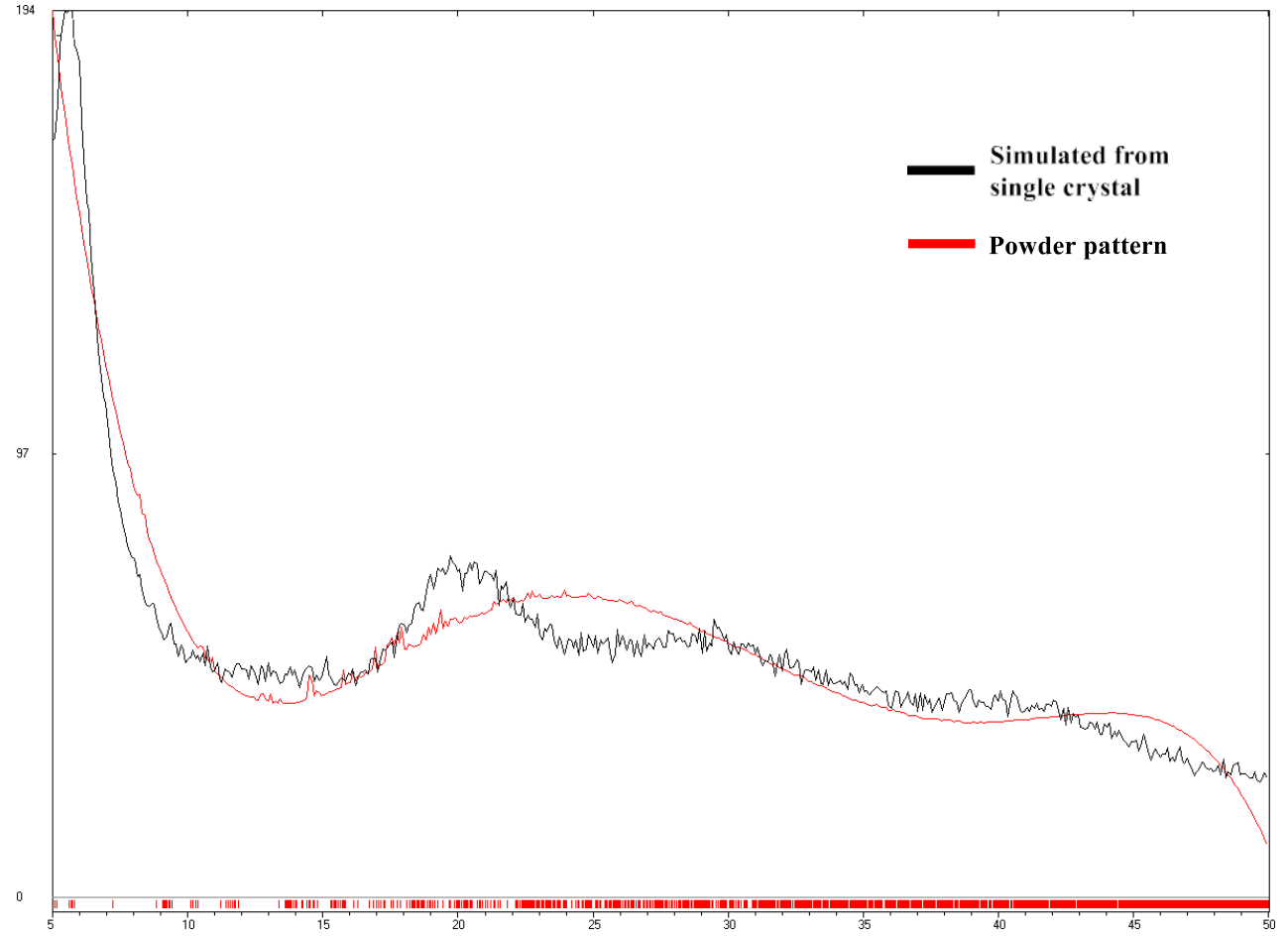

Figure S19: Powder X-ray diffraction pattern of a bulk sample of $\mathbf{3}$ compared to the simulated powder pattern extracted from single crystal diffraction data.

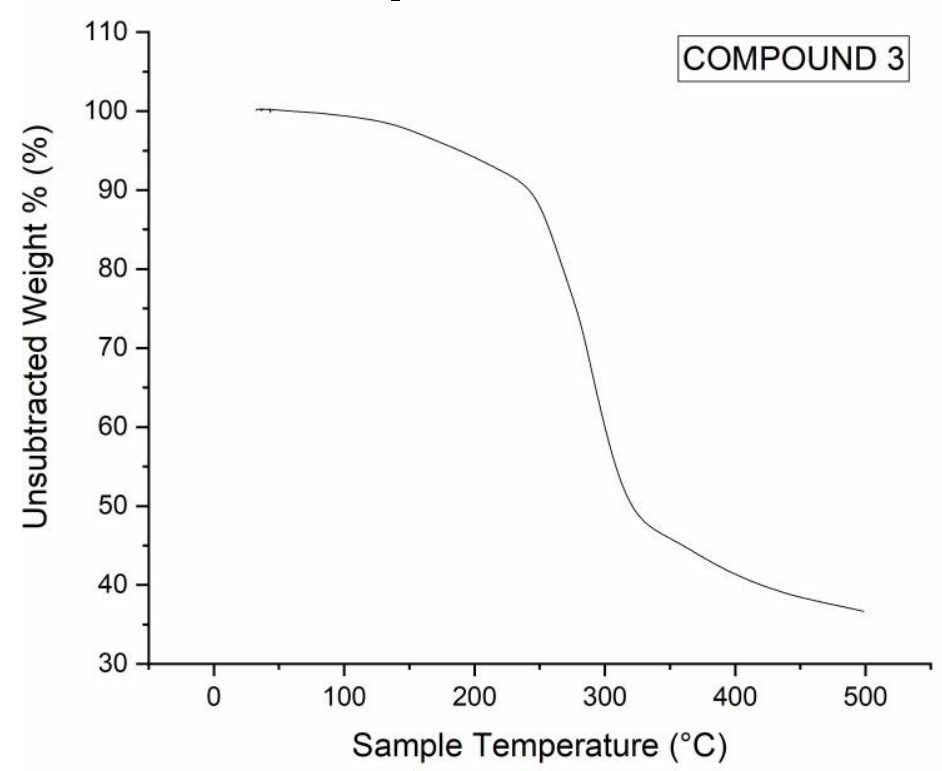

Figure S20: The TGA curve of 3 heating to $500{ }^{\circ} \mathrm{C}$ in $\mathrm{N}_{2}$ at a rate of $10{ }^{\circ} \mathrm{C}$ min. 


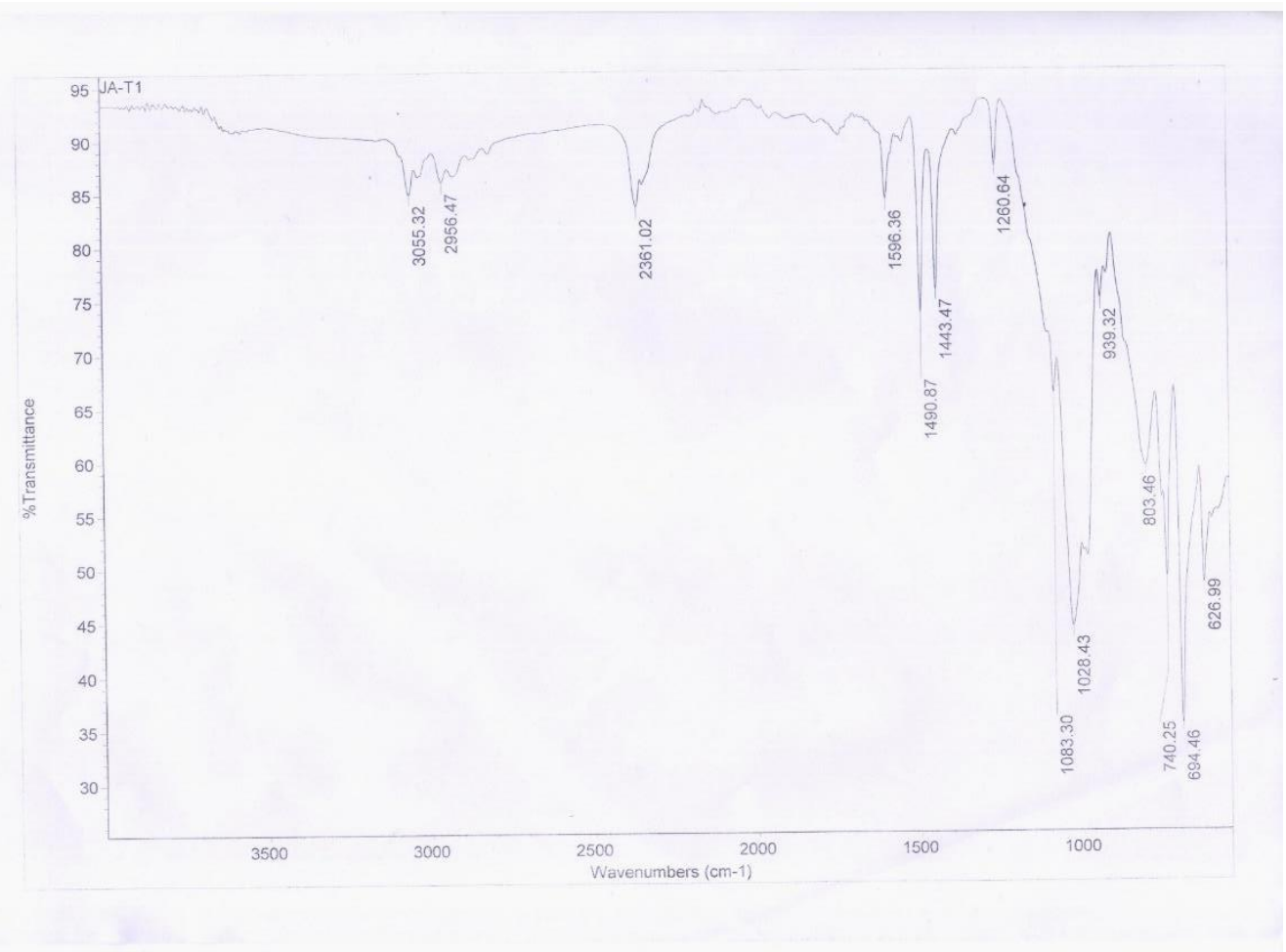

Figure S21: IR spectra of compound 3. $3055 \mathrm{~cm}^{-1}$ (aromatic C-H stretching), $2361 \mathrm{~cm}^{-1}$ (P-H stretching), $1083 \mathrm{~cm}^{-1}, 1028 \mathrm{~cm}^{-1}$ (P-O-M stretching).
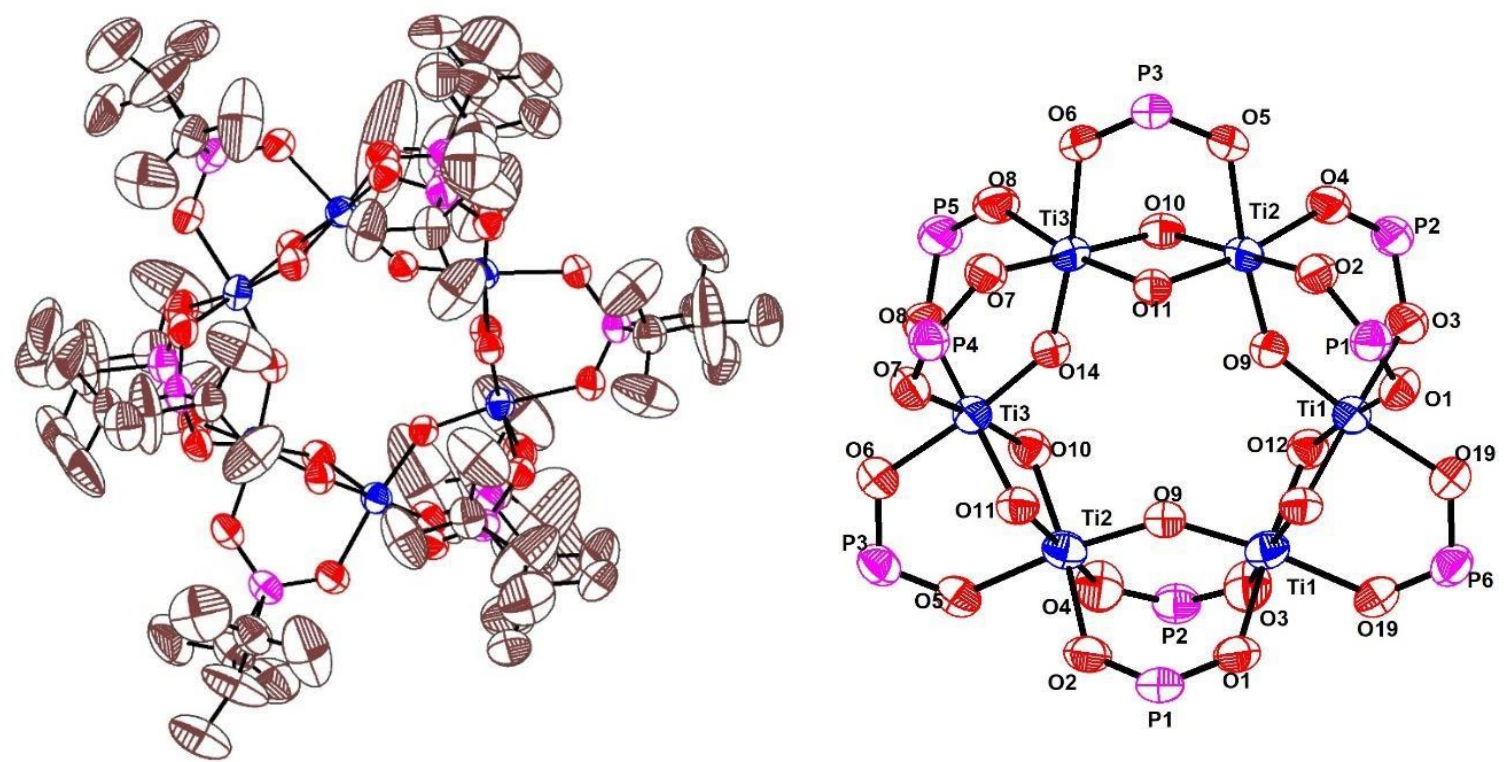

Figure S22: ORTEP view of $\mathbf{4}$ and metal oxo core with thermal ellipsoids shown at 30\% Probability. 
Table S5: Selected bond lengths $(\AA)$ and bond angles (deg) parameters of compound 4.

\begin{tabular}{|c|c|c|}
\hline $\mathrm{Ti}(1)-\mathrm{O}(12) \quad 1.941(2)$ & $\mathrm{O}(9)-\mathrm{Ti}(1)-\mathrm{O}(13) \quad 95.73(13)$ & $\mathrm{O}(4)-\mathrm{Ti}(2)-\mathrm{O}(5) \quad 87.60(11)$ \\
\hline $\mathrm{Ti}(1)-\mathrm{O}(9)$ & $\mathrm{O}(9)-\mathrm{Ti}(1)-\mathrm{O}(19)$ & $\mathrm{O}(2)-\mathrm{Ti}(2)-\mathrm{O}(5)$ \\
\hline $\mathrm{Ti}(1)-\mathrm{O}(13)$ & $\mathrm{O}(9)-\mathrm{Ti}(1)-\mathrm{O}(3)$ & $\mathrm{O}(10)-\mathrm{Ti}(3)-\mathrm{O}(11) 80.15(11)$ \\
\hline $\mathrm{Ti}(1)-\mathrm{O}(19)$ & $\mathrm{O}(9)-\mathrm{Ti}(1)-\mathrm{O}(1)$ & $\mathrm{O}(10)-\mathrm{Ti}(3)-\mathrm{O}(8) \quad 94.13(12)$ \\
\hline $\operatorname{Ti}(1)-\mathrm{O}(3)$ & $\mathrm{O}(13)-\mathrm{Ti}(1)-\mathrm{O}(19) 88.85(13)$ & $\mathrm{O}(10)-\mathrm{Ti}(3)-\mathrm{O}(7) \quad 169.82(13)$ \\
\hline $\operatorname{Ti}(1)-\mathrm{O}(1)$ & $\mathrm{O}(13)-\mathrm{Ti}(1)-\mathrm{O}(1)$ & $\mathrm{O}(10)-\mathrm{Ti}(3)-\mathrm{O}(6)$ \\
\hline $\mathrm{Ti}(2)-\mathrm{O}(9)$ & $\mathrm{O}(13)-\mathrm{Ti}(1)-\mathrm{Ti}(1)^{1} \quad 40.52(7)$ & $\mathrm{O}(14)-\mathrm{Ti}(3)-\mathrm{O}(10)$ 96.79(14) \\
\hline $\mathrm{Ti}(2)-\mathrm{O}(10)$ & $\mathrm{O}(3)-\mathrm{Ti}(1)-\mathrm{O}(19) \quad 86.92(10)$ & $\mathrm{O}(14)-\mathrm{Ti}(3)-\mathrm{O}(11) 96.14(13)$ \\
\hline $\mathrm{Ti}(2)-\mathrm{O}(11)$ & $\mathrm{O}(1)-\mathrm{Ti}(1)-\mathrm{O}(19)$ & $\mathrm{O}(14)-\mathrm{Ti}(3)-\mathrm{O}(8)$ \\
\hline $\mathrm{Ti}(2)-\mathrm{O}(4)$ & $\mathrm{O}(1)-\mathrm{Ti}(1)-\mathrm{O}(3)$ & $\mathrm{O}(14)-\mathrm{Ti}(3)-\mathrm{O}(7) \quad 89.86(15)$ \\
\hline $\mathrm{Ti}(2)-\mathrm{O}(2)$ & $\mathrm{O}(9)-\mathrm{Ti}(2)-\mathrm{O}(10) \quad 94.88(11)$ & $\mathrm{O}(14)-\mathrm{Ti}(3)-\mathrm{O}(6)$ \\
\hline $\mathrm{Ti}(2)-\mathrm{O}(5)$ & $\mathrm{O}(9)-\mathrm{Ti}(2)-\mathrm{O}(11) \quad 94.49(11)$ & $\mathrm{O}(11)-\mathrm{Ti}(3)-\mathrm{O}(8)$ \\
\hline $\mathrm{Ti}(3)-\mathrm{O}(10)$ & $\mathrm{O}(9)-\mathrm{Ti}(2)-\mathrm{O}(4)$ & $\mathrm{O}(11)-\mathrm{Ti}(3)-\mathrm{O}(7) \quad 91.51(13)$ \\
\hline $\mathrm{Ti}(3)-\mathrm{O}(14)$ & $\mathrm{O}(9)-\mathrm{Ti}(2)-\mathrm{O}(2)$ & $\mathrm{O}(11)-\mathrm{Ti}(3)-\mathrm{O}(6) \quad 89.05(12)$ \\
\hline $\mathrm{Ti}(3)-\mathrm{O}(11)$ & $\mathrm{O}(9)-\mathrm{Ti}(2)-\mathrm{O}(5)$ & $\mathrm{O}(8)-\mathrm{Ti}(3)-\mathrm{O}(6)$ \\
\hline $\mathrm{Ti}(3)-\mathrm{O}(8)$ & $\mathrm{O}(10)-\mathrm{Ti}(2)-\mathrm{O}(11) \quad 80.77(11)$ & $\mathrm{O}(7)-\mathrm{Ti}(3)-\mathrm{O}(8)$ \\
\hline $\mathrm{Ti}(3)-\mathrm{O}(7)$ & $\mathrm{O}(10)-\mathrm{Ti}(2)-\mathrm{O}(4) \quad 95.65(12)$ & $\mathrm{O}(7)-\mathrm{Ti}(3)-\mathrm{O}(6)$ \\
\hline $\mathrm{Ti}(3)-\mathrm{O}(6)$ & $\mathrm{O}(10)-\mathrm{Ti}(2)-\mathrm{O}(2)$ & $\operatorname{Ti}\left(1^{1}\right)-\mathrm{O}(12)-\mathrm{Ti}(1) \quad 100.49(15)$ \\
\hline $\mathrm{O}(12)-\mathrm{Ti}(1)-\mathrm{O}(13) 79.93(11)$ & $\mathrm{O}(10)-\mathrm{Ti}(2)-\mathrm{O}(5)$ & $\operatorname{Ti}(1)-\mathrm{O}(9)-\operatorname{Ti}(2)$ \\
\hline $\mathrm{O}(12)-\mathrm{Ti}(1)-\mathrm{O}(19) 87.97(12)$ & $\mathrm{O}(11)-\mathrm{Ti}(2)-\mathrm{O}(4)$ & $\mathrm{Ti}(2)-\mathrm{O}(10)-\mathrm{Ti}(3) \quad 101.42(12)$ \\
\hline $\mathrm{O}(12)-\mathrm{Ti}(1)-\mathrm{O}(3) 92.95(11)$ & $\mathrm{O}(11)-\mathrm{Ti}(2)-\mathrm{O}(2)$ & $\operatorname{Ti}\left(3^{1}\right)-\mathrm{O}(14)-\operatorname{Ti}(3) \quad 143.3(2)$ \\
\hline $\mathrm{O}(12)-\mathrm{Ti}(1)-\mathrm{O}(1) \quad 170.48(12)$ & $\mathrm{O}(11)-\mathrm{Ti}(2)-\mathrm{O}(5)$ & $\operatorname{Ti}\left(1^{1}\right)-\mathrm{O}(13)-\mathrm{Ti}(1) \quad 99.04(15)$ \\
\hline $\mathrm{O}(9)-\mathrm{Ti}(1)-\mathrm{O}(12) 97.11(13)$ & $\mathrm{O}(4)-\mathrm{Ti}(2)-\mathrm{O}(2)$ & $\mathrm{Ti}(2)-\mathrm{O}(11)-\mathrm{Ti}(3) \quad 96.88(11)$ \\
\hline
\end{tabular}




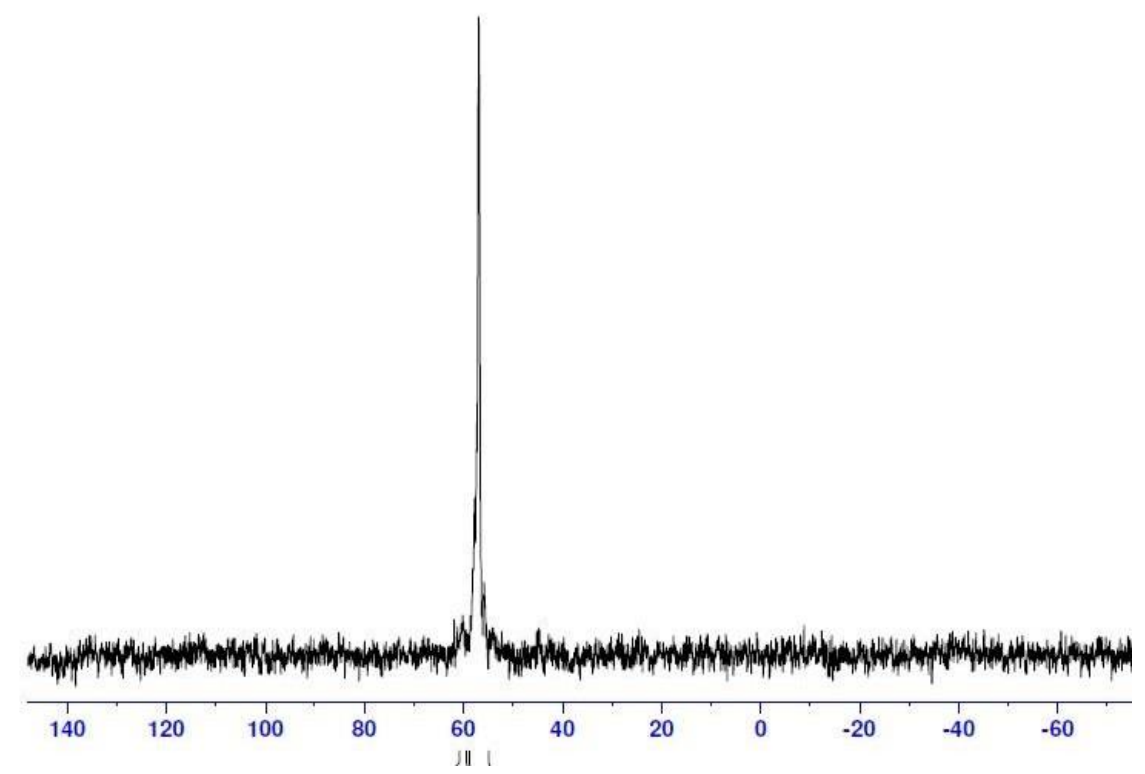

Figure S23: ${ }^{31} \mathbf{P}$ NMR $\left(162 \mathrm{MHz}, \mathrm{CDCl}_{3}\right)$ of compound 4 at room temperature.

$$
\text { ָั }
$$

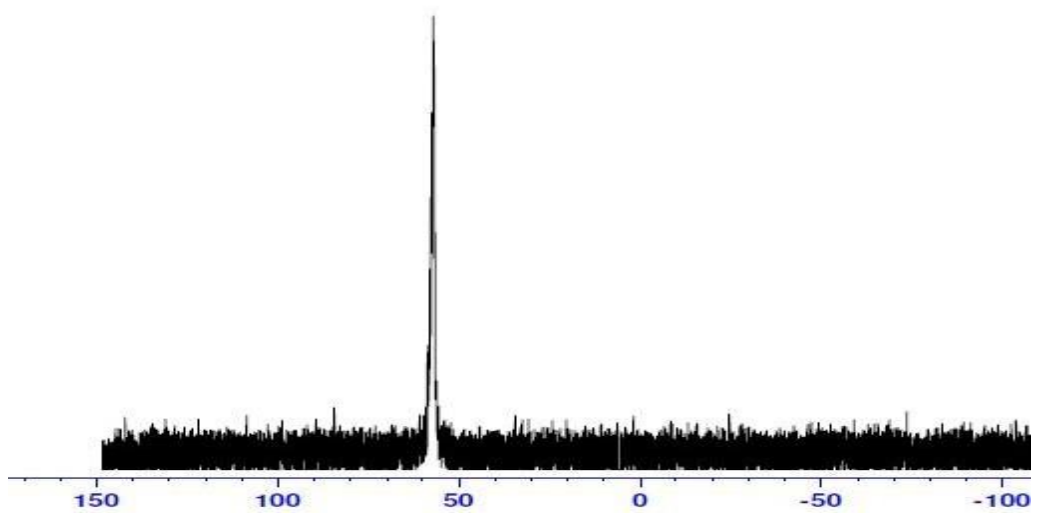

Figure S24: ${ }^{31} \mathbf{P}$ NMR $\left(162 \mathrm{MHz}, \mathrm{CDCl}_{3}\right)$ of compound 4 at $0^{\circ} \mathrm{C}$. 


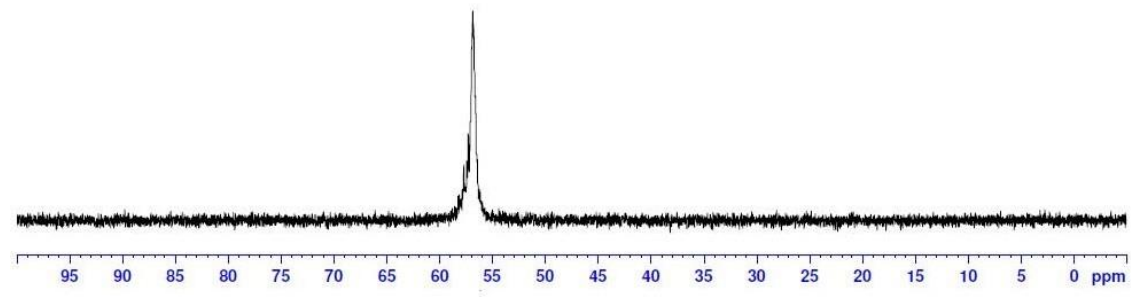

Figure S25: ${ }^{31} \mathrm{P}$ NMR $\left(162 \mathrm{MHz}, \mathrm{CDCl}_{3}\right)$ of compound 4 at $-10^{\circ} \mathrm{C}$.

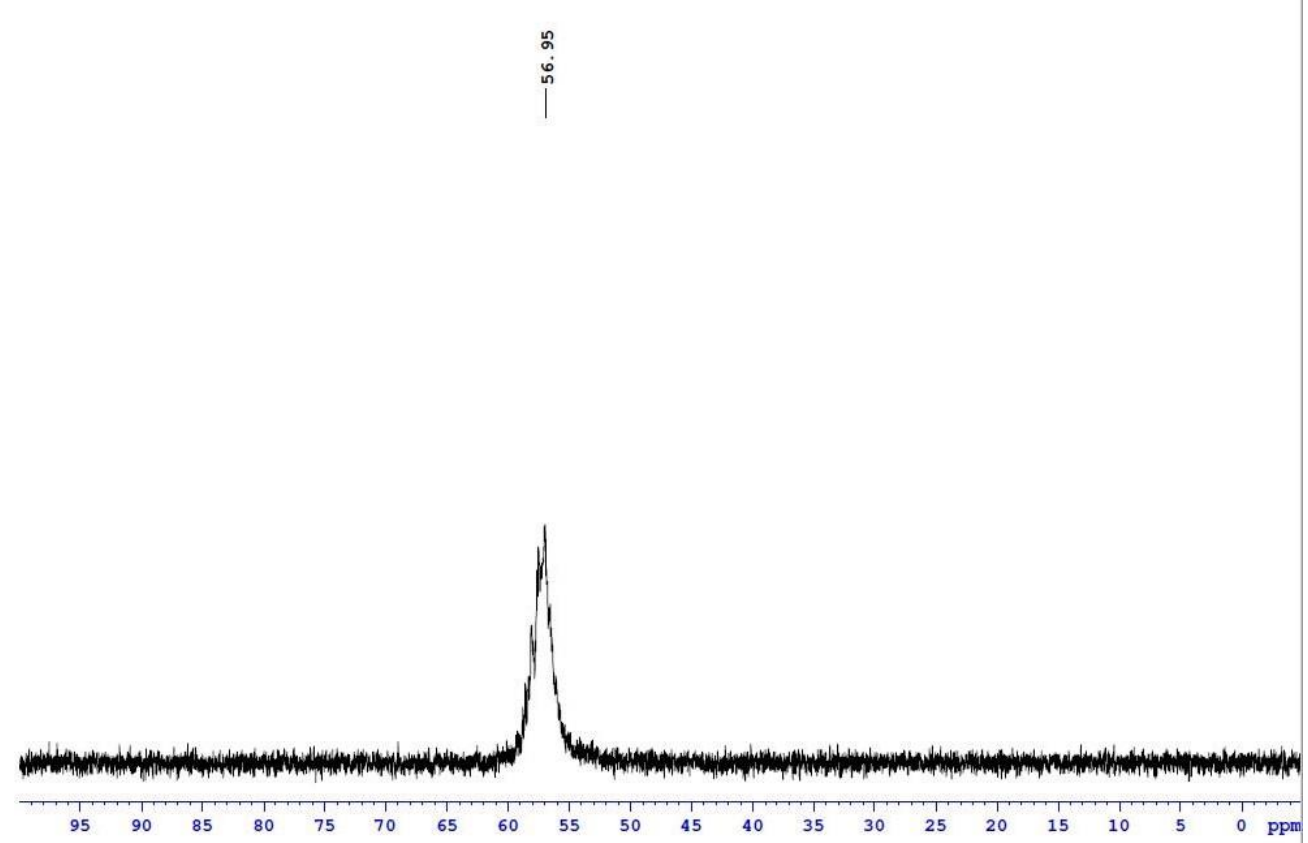

Figure S26: ${ }^{31} \mathbf{P}$ NMR $\left(162 \mathrm{MHz}, \mathrm{CDCl}_{3}\right)$ of compound $\mathbf{4}$ at $-20^{\circ} \mathrm{C}$. 


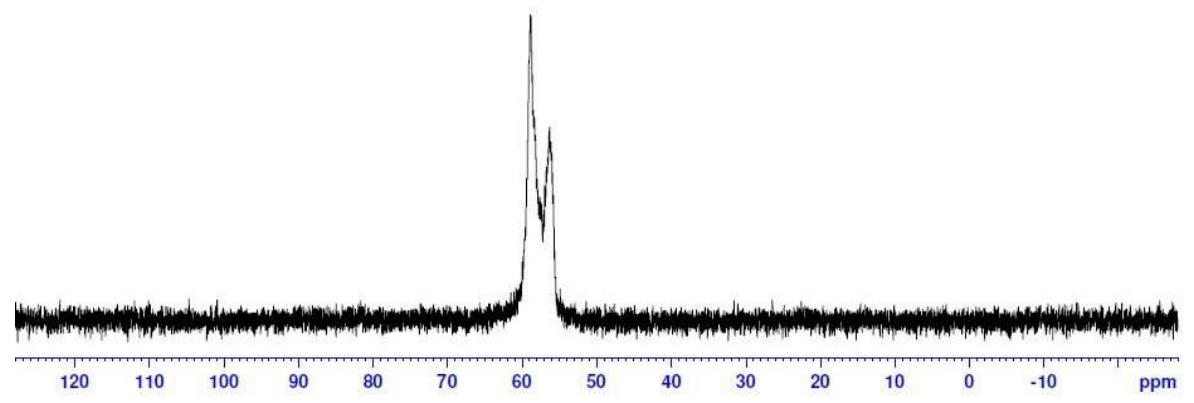

Figure S27: ${ }^{31} \mathbf{P}$ NMR $\left(162 \mathrm{MHz}, \mathrm{CDCl}_{3}\right)$ of compound 4 at $-30^{\circ} \mathrm{C}$.

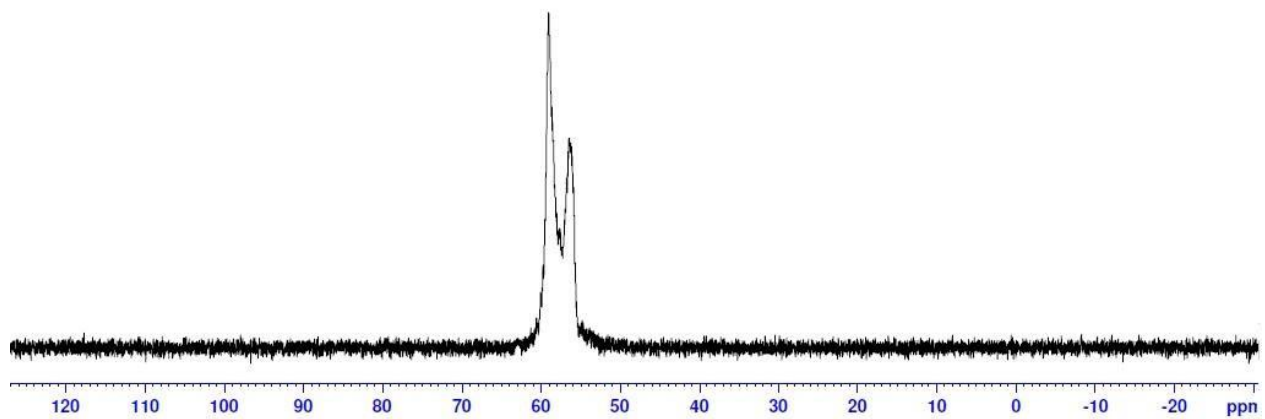

Figure S28: ${ }^{31} \mathbf{P}$ NMR $\left(162 \mathrm{MHz}, \mathrm{CDCl}_{3}\right)$ of compound $\mathbf{4}$ at $-40^{\circ} \mathrm{C}$. 

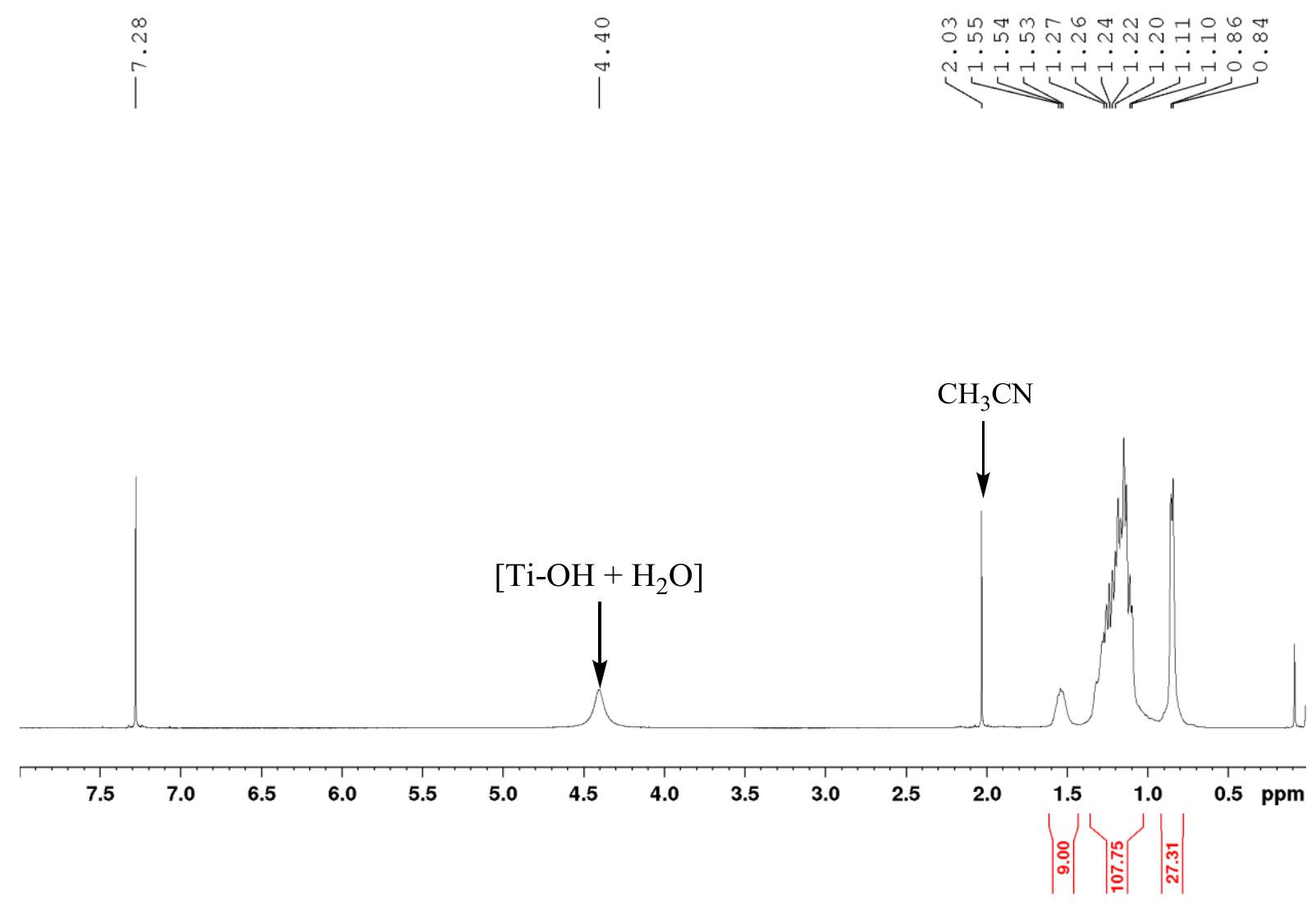

Figure S29: ${ }^{1} \mathbf{H}$ NMR $\left(500 \mathrm{MHz}, \mathrm{CDCl}_{3}\right)$ of compound 4.

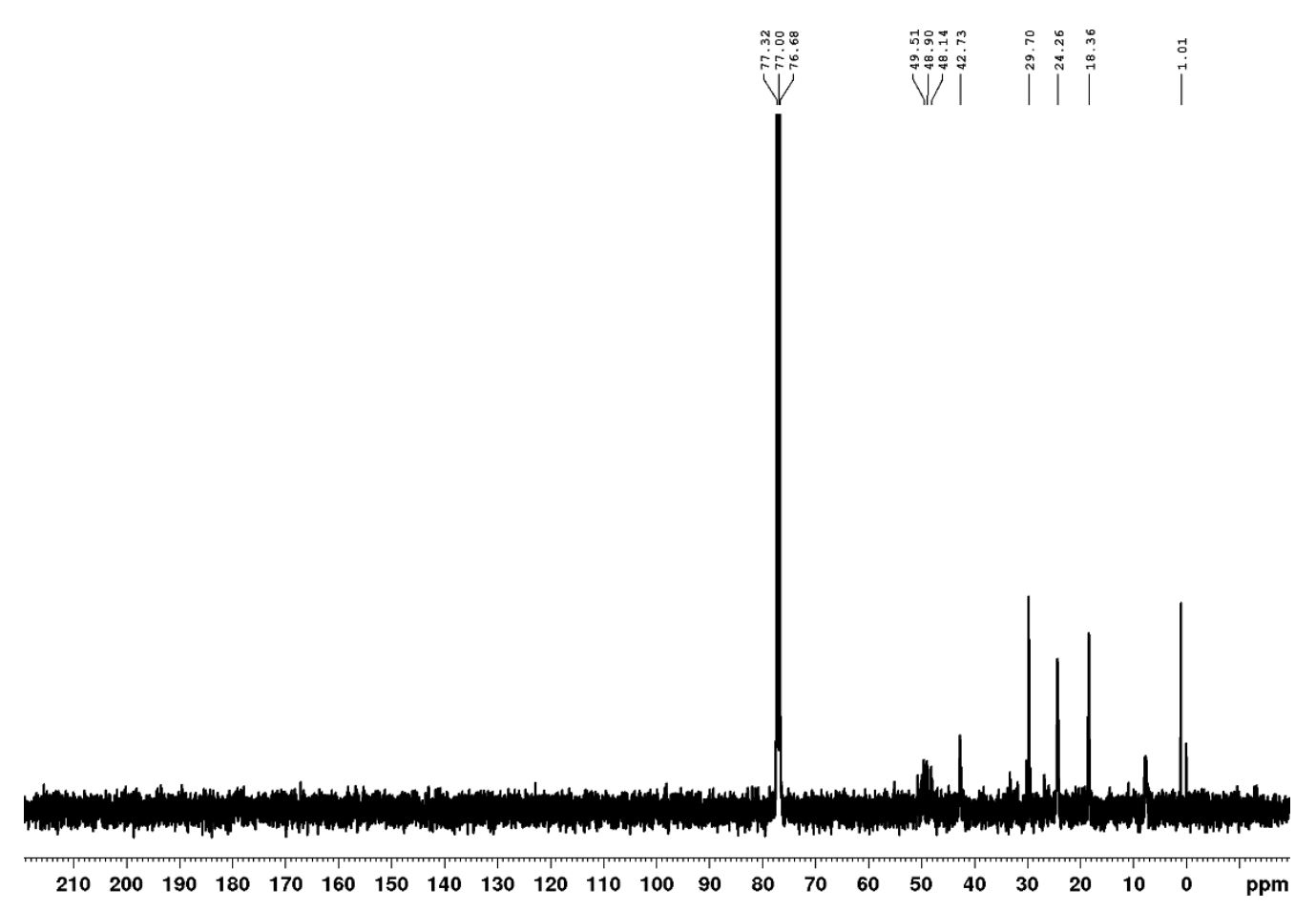

Figure S30: ${ }^{13} \mathrm{C}$ NMR $\left(100 \mathrm{MHz}, \mathrm{CDCl}_{3}\right)$ of compound 4. 


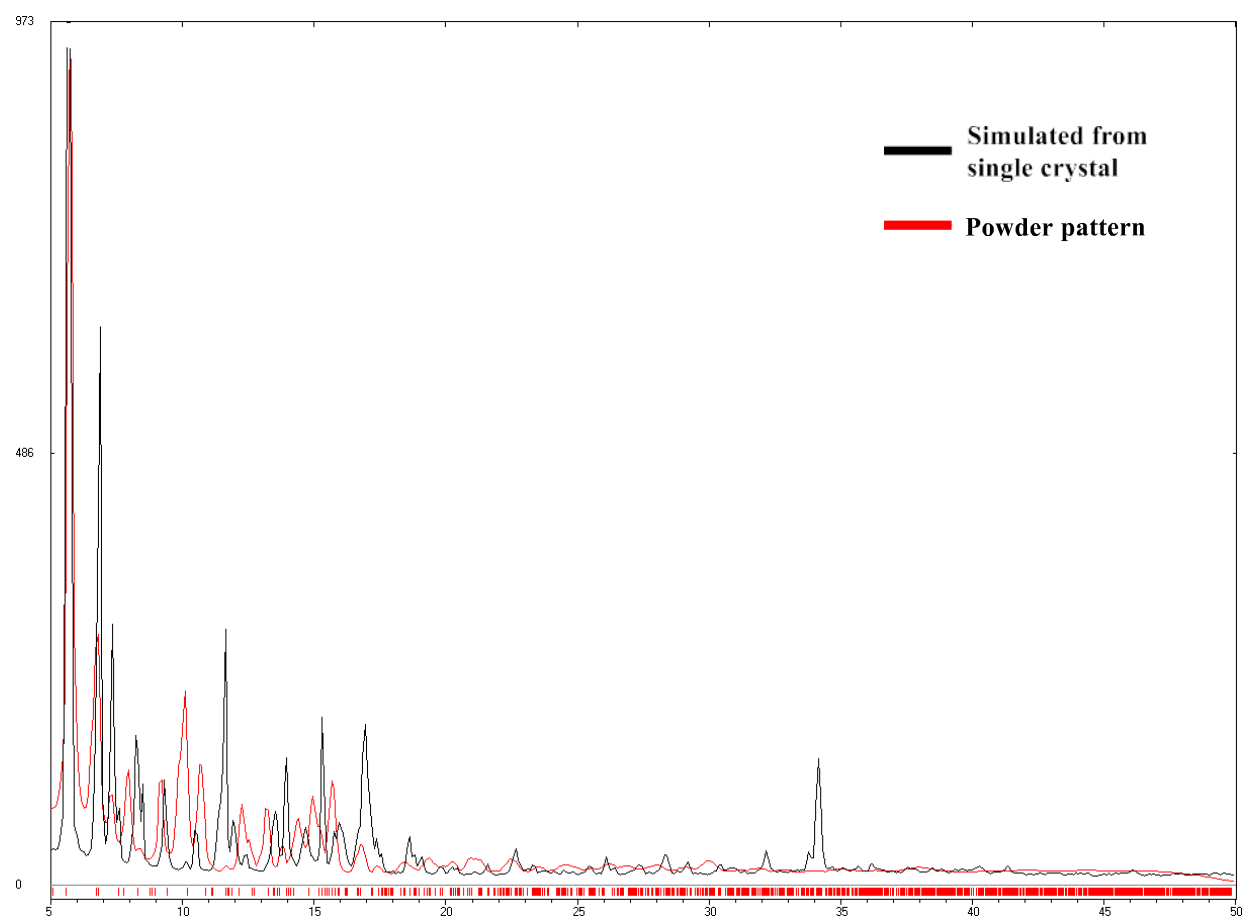

Figure S31: Powder X-ray diffraction pattern of a bulk sample of $\mathbf{4}$ compared to the simulated powder pattern extracted from single crystal diffraction data.

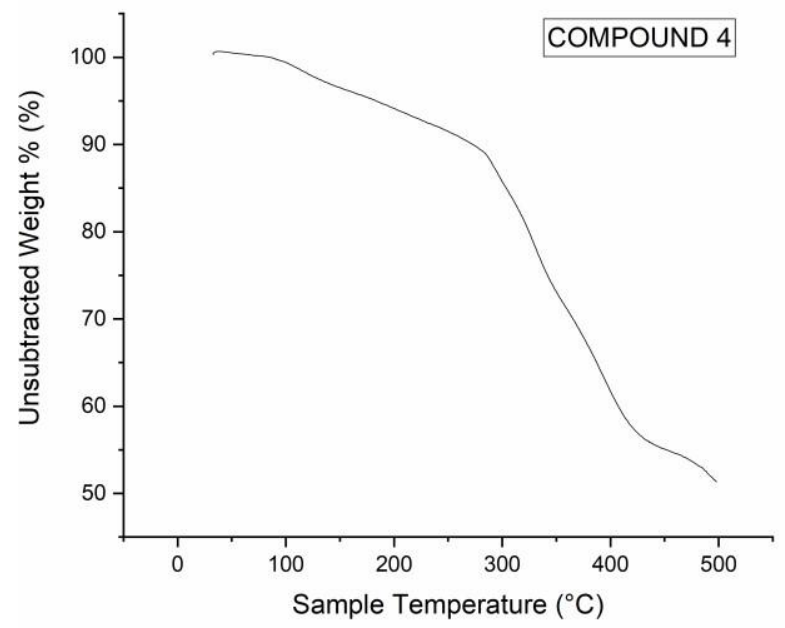

Figure S32: The TGA curve of 4 heating to $500{ }^{\circ} \mathrm{C}$ in $\mathrm{N} 2$ at a rate of $10{ }^{\circ} \mathrm{C}$ min. 


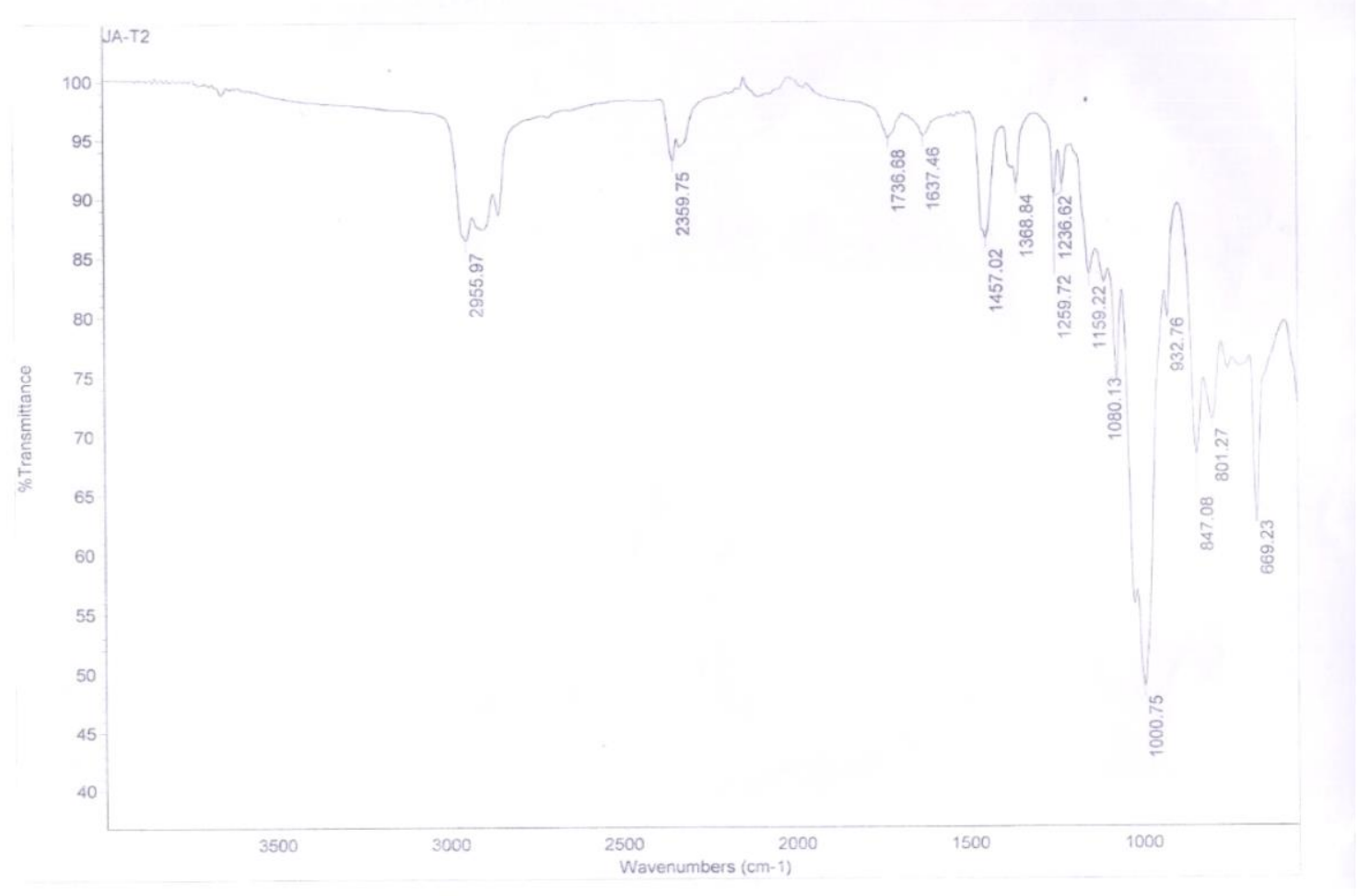

Figure S33: IR spectra of compound 4. $3055 \mathrm{~cm}^{-1}$ (C-H stretching), $2361 \mathrm{~cm}^{-1}, 1124 \mathrm{~cm}^{-1}, 1082$ $\mathrm{cm}^{-1}, 1015 \mathrm{~cm}^{-1}$ (P-O-M stretching).

Table S6: Shape calculation of Bi ions in compound 1.

S H A P E v2.1 Continuous Shape Measures calculation

(c) 2013 Electronic Structure Group, Universitat de Barcelona

Contact: llunell@ub.edu

\section{J_Bi-1}

PP-5 D5h Pentagon

vOC-5 2 C4v Vacant octahedron TBPY-

53 D3h Trigonal bipyramid SPY-

$54 \mathrm{C4v}$ Spherical square pyramid

JTBPY-5 5 D3h Johnson trigonal bipyramid J12

$\begin{array}{llllll}\text { Structure [ML5 ] } & \text { PP-5 } & \text { vOC-5 } & \text { TBPY-5 } & \text { SPY-5 } & \text { JTBPY-5 }\end{array}$

Bi

35.337, 5.946, $0.166, \quad 3.963, \quad 2.825$


Table S7: Shape calculation of Bi ions in compound 2.

J_Bi-1

$\begin{array}{llllll}\text { Structure [ML5 ] } & \text { PP-5 } & \text { vOC-5 } & \text { TBPY-5 } & \text { SPY-5 } & \text { JTBPY-5 }\end{array}$

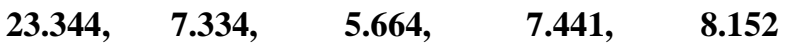

J_Bi-2

$\begin{array}{llllll}\text { Structure [ML5 ] } & \text { PP-5 } & \text { vOC-5 } & \text { TBPY-5 } & \text { SPY-5 } & \text { JTBPY-5 }\end{array}$

28.256, 2.137, $11.565, \quad 6.080, \quad 11.250$

J_Bi-3

$\begin{array}{llllll}\text { Structure [ML5 ] } & \text { PP-5 } & \text { vOC-5 } & \text { TBPY-5 } & \text { SPY-5 } & \text { JTBPY-5 }\end{array}$

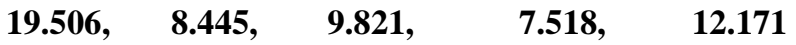

J_Bi-4

Structure [ML5 ] $\quad$ PP-5 $\quad$ vOC-5 TBPY-5 SPY-5 $\quad$ JTBPY-5

$27.89, \quad 2.064, \quad 10.628, \quad 5.956, \quad 10.841$

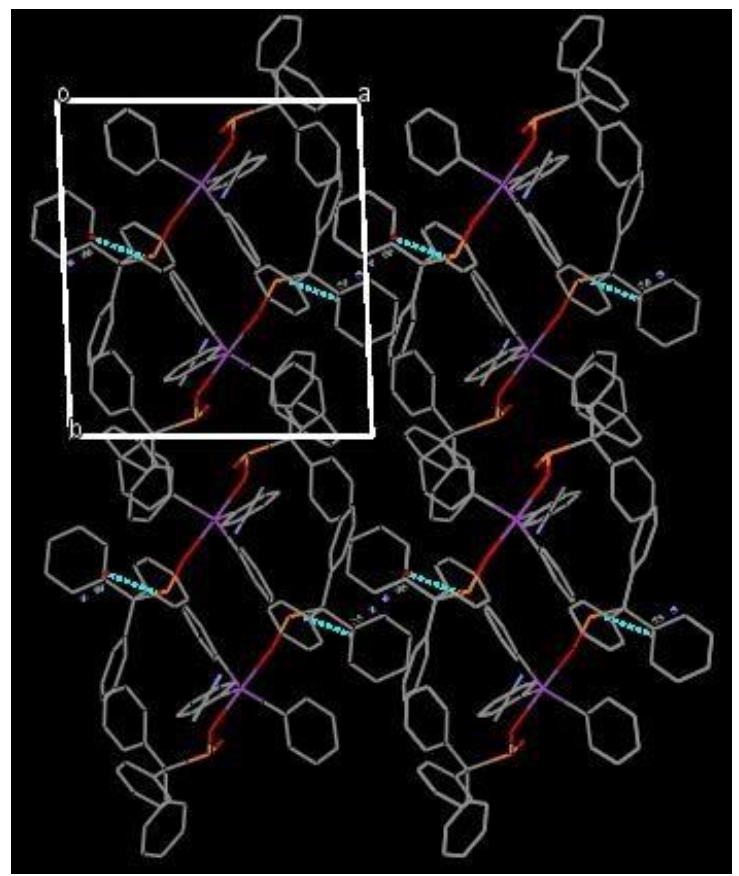

Figure S34: Packing diagram of compound 1. 


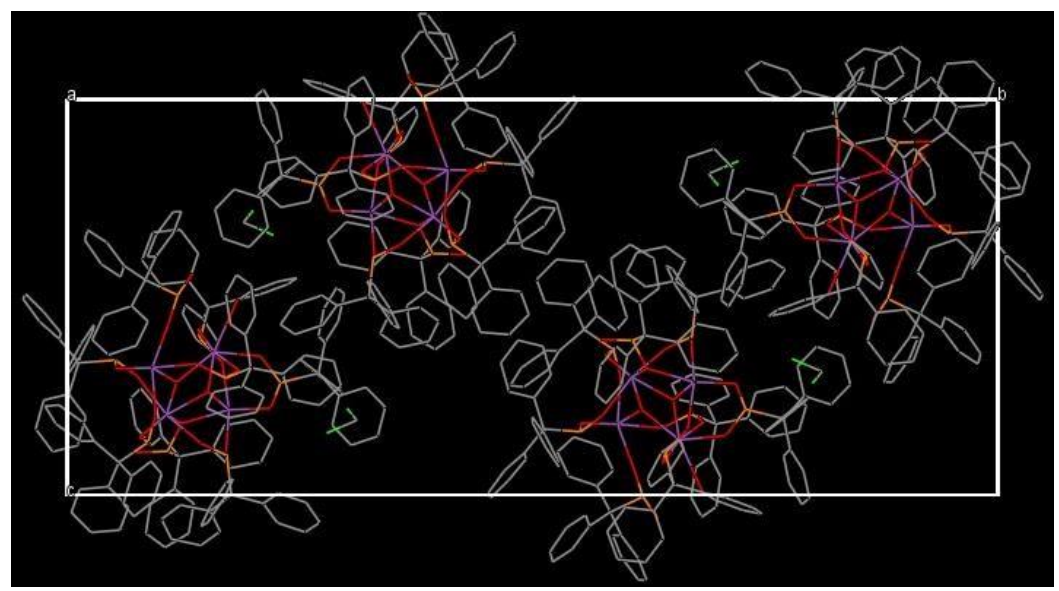

Figure S35: Packing diagram of compound 2.

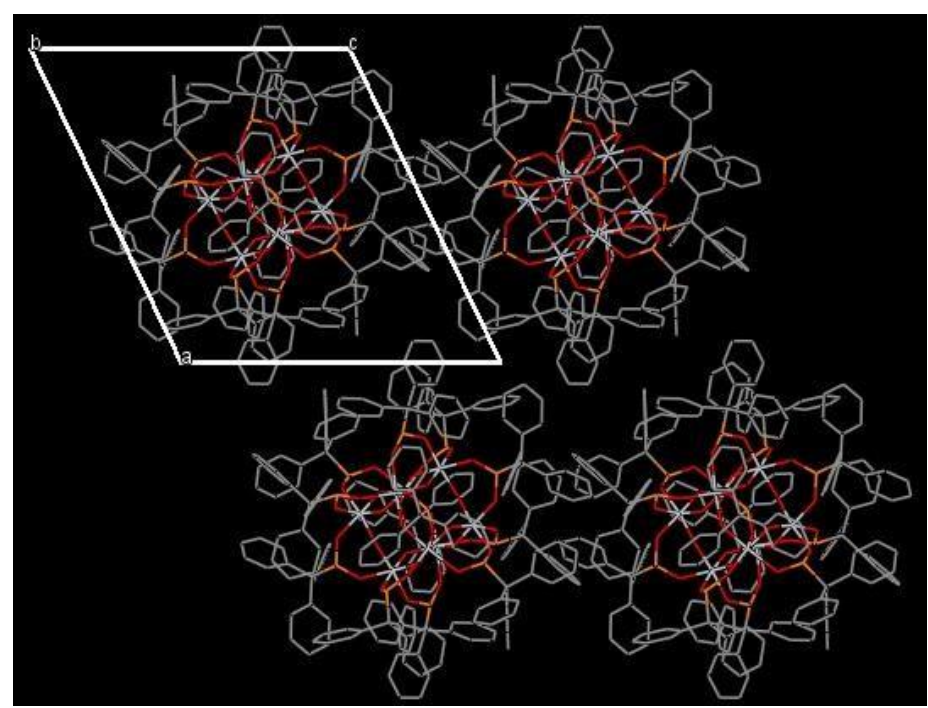

Figure S36: Packing diagram of compound 3.

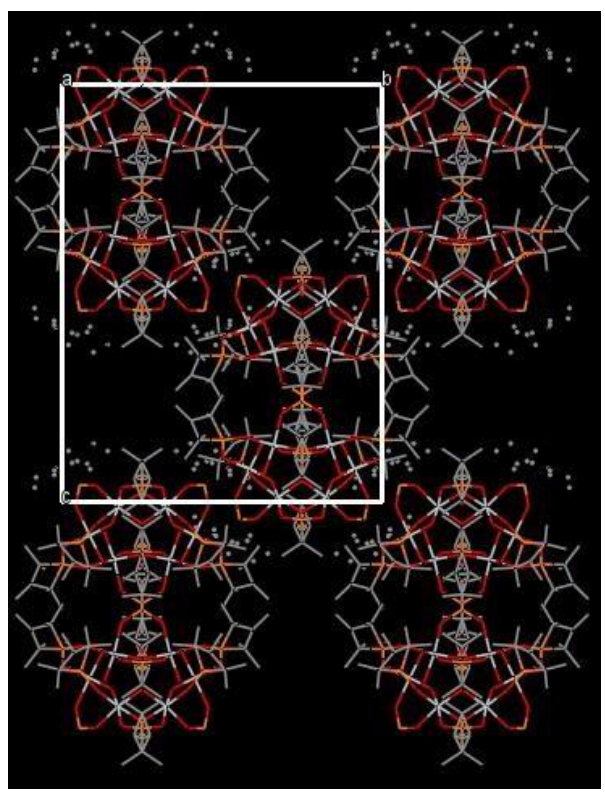

Figure S37: Packing diagram of compound 4. 


\section{Solvent treatment of compounds 2-4.}

Compound 2: For 2 A solvent mask was calculated and 256 electrons were found in a volume of $1026 \backslash \% \mathrm{~A}^{3}$ in 3 voids. This is consistent with the presence of $1\left[\mathrm{CH}_{2} \mathrm{Cl}_{2}\right], 0.5\left[\mathrm{C}_{2} \mathrm{H}_{3} \mathrm{~N}\right], 1\left[\mathrm{H}_{2} \mathrm{O}\right]$ per formula unit which account for 252.0 electrons. $[(4 \times 42)+(4 \times 11)+(4 \times 10)=252]$

Compound 3: A solvent mask was calculated and 165 electrons were found in a volume of $1440 \backslash \% \mathrm{~A}^{3}$ in 1 void. This is consistent with the presence of $3\left[\mathrm{C}_{7} \mathrm{H}_{8}\right]$ per formula unit which account for 150.0 electrons.

Compound 3: A solvent mask was calculated and 417 electrons were found in a volume of $26161 \% \mathrm{~A}^{3}$ in 2 voids. This is consistent with the presence of $4\left[\mathrm{C}_{2} \mathrm{H}_{3} \mathrm{~N}\right], 2\left[\mathrm{H}_{2} \mathrm{O}\right]$, per formula unit which account for 432.0 electrons. [ $(4 \times 88)+(4 \times 20)=432]$

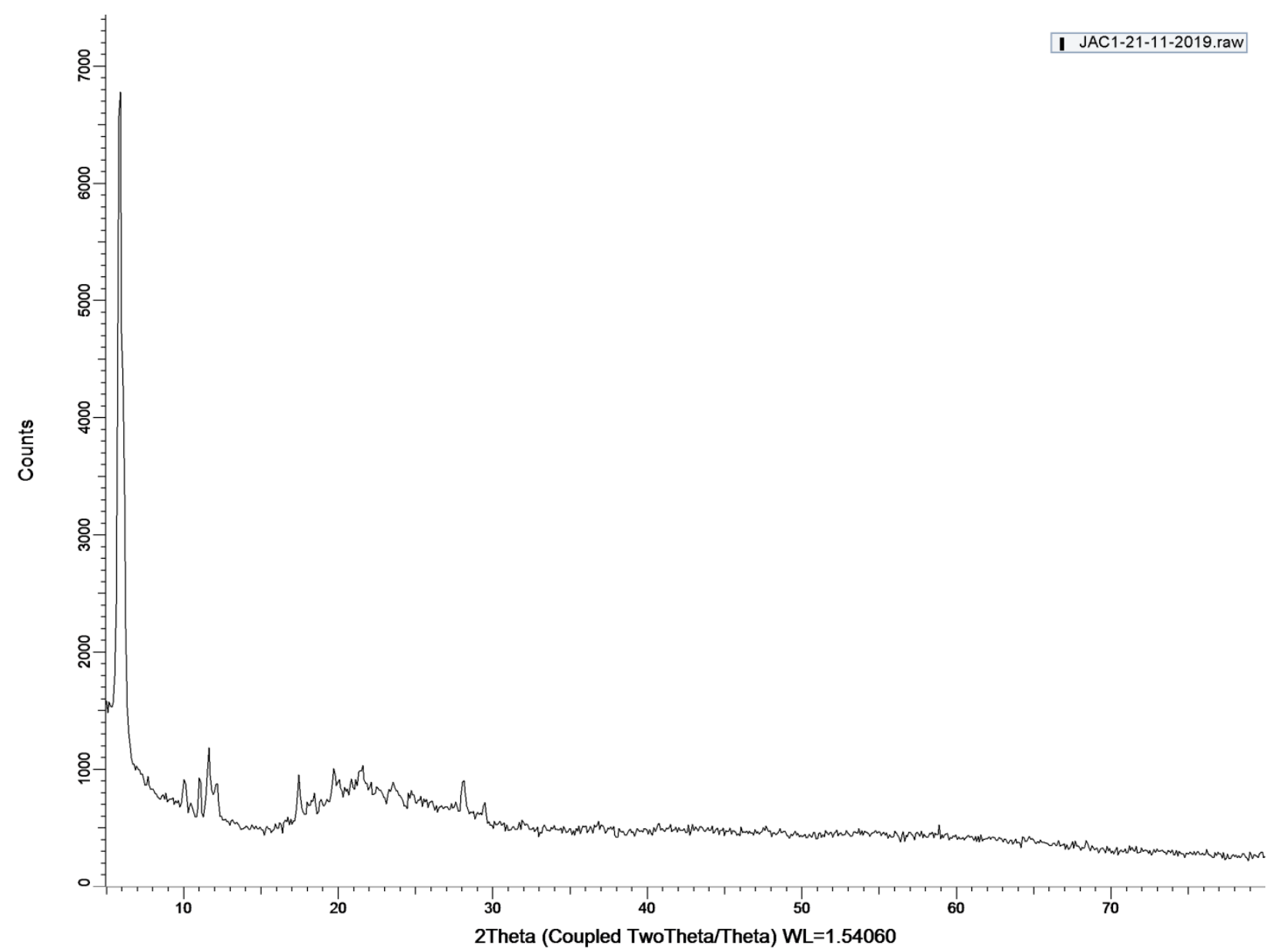

Figure S38: Powder X-ray diffraction pattern of the residue sample of $\mathbf{1 .}$ 


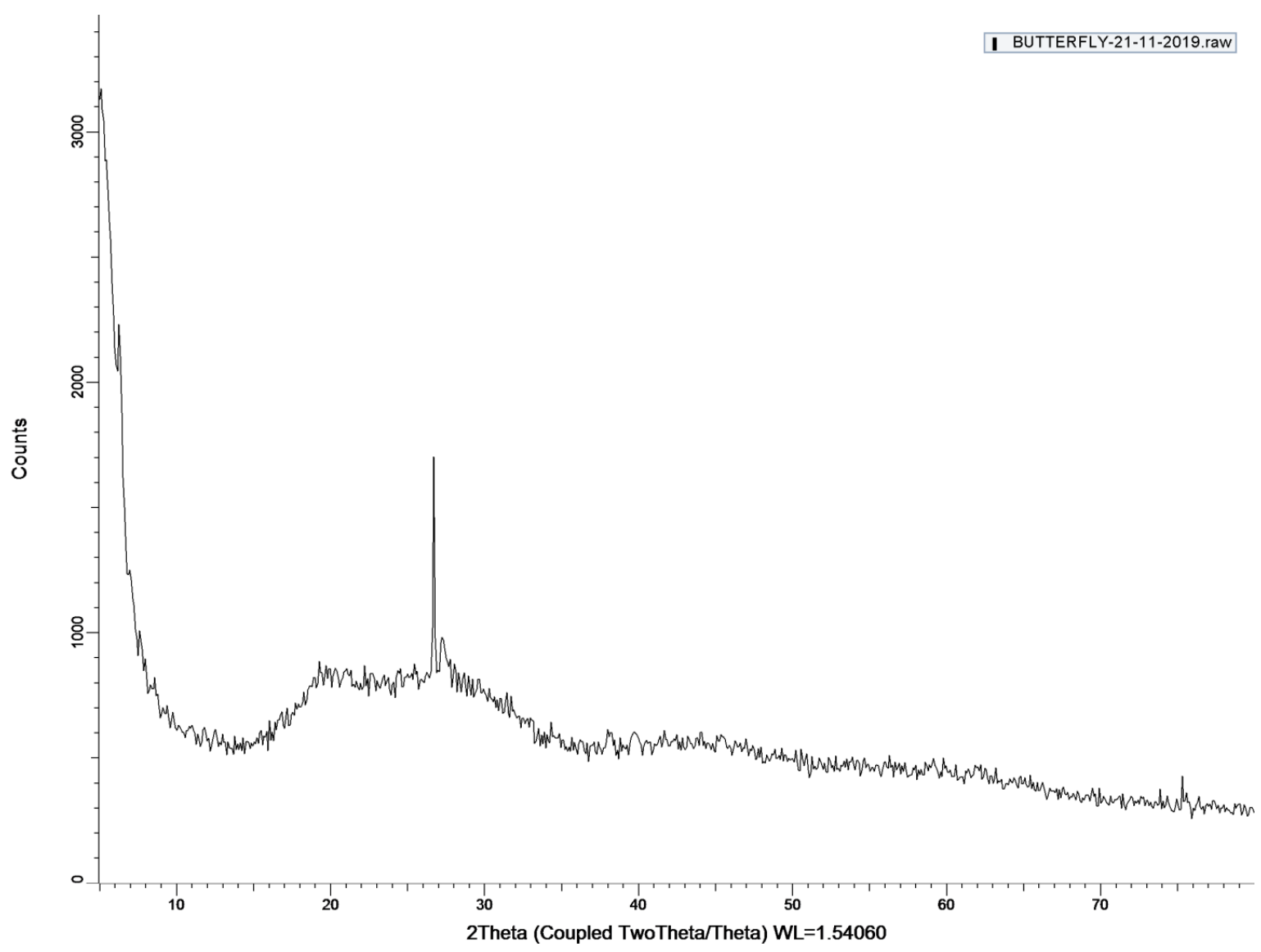

Figure S39: Powder X-ray diffraction pattern of the residue sample of 2.

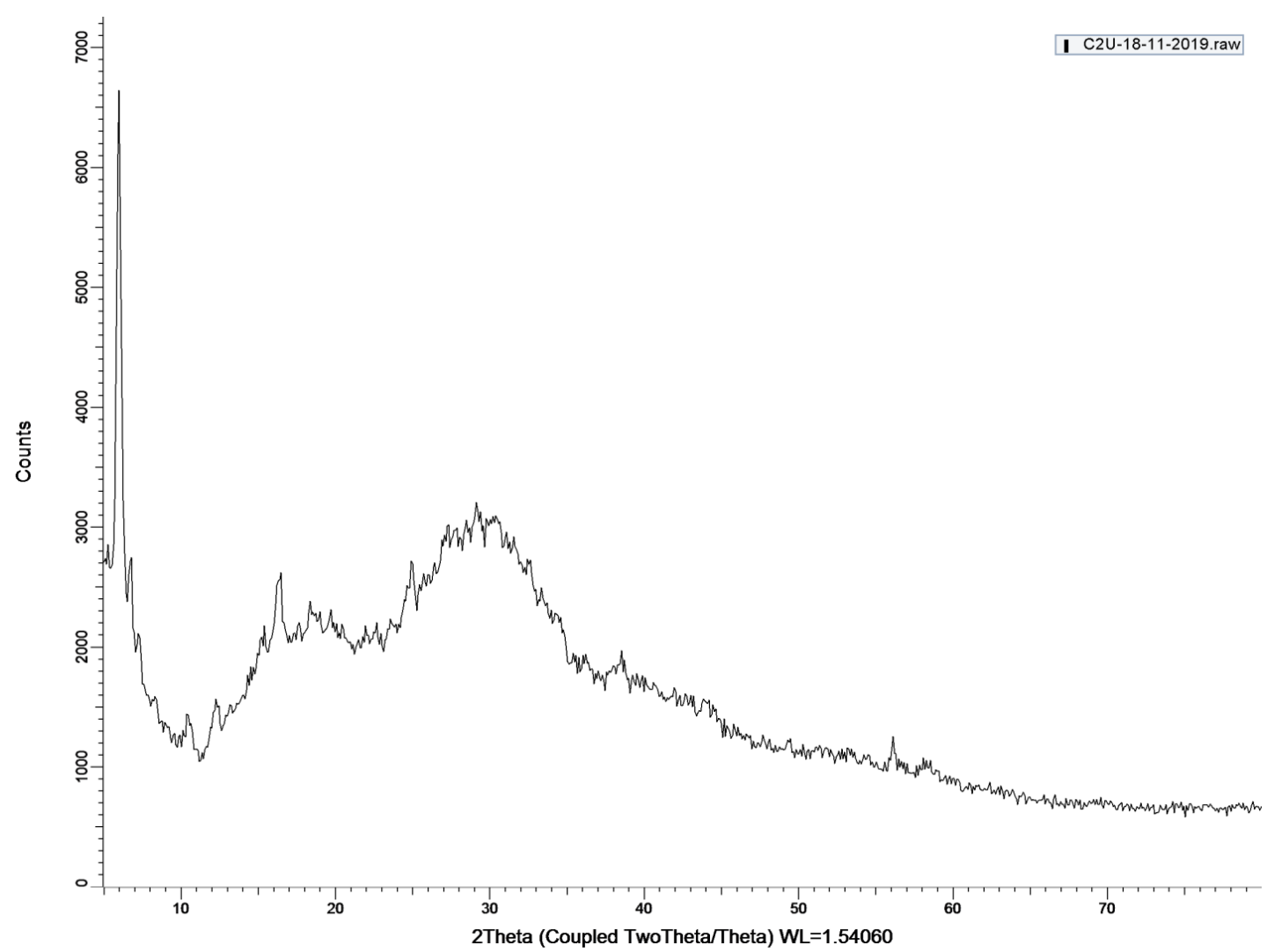

Figure S40: Powder X-ray diffraction pattern of the residue sample of $\mathbf{3}$. 


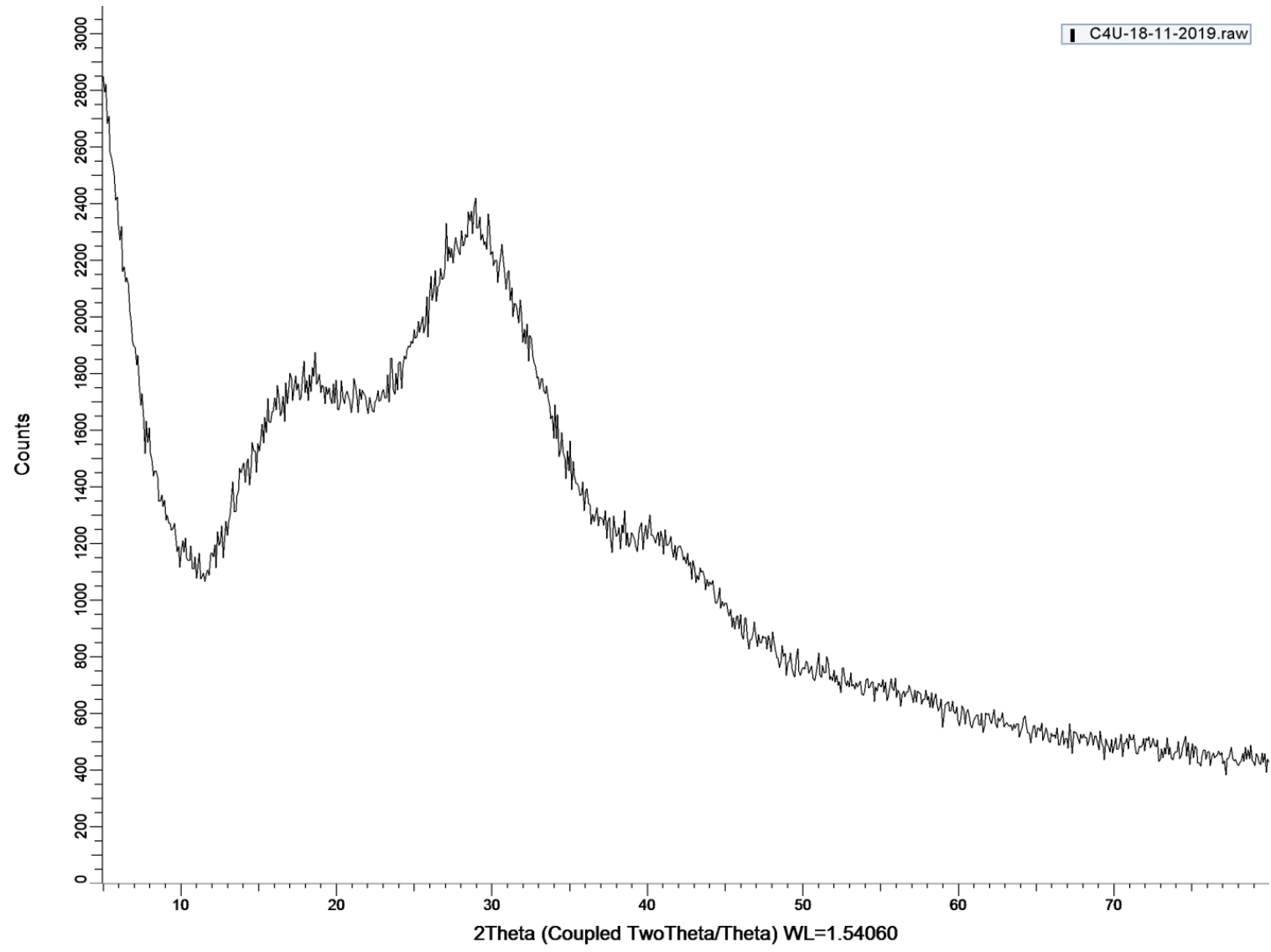

Figure S41: Powder X-ray diffraction pattern of the residue sample of 3. 